\title{
Vorticity-pressure formulations for the Brinkman-Darcy coupled problem
}

\author{
Verónica Anaya \\ GIMNAP, Departamento de Matemática, Universidad del Bío-Bío, Concepción, Chile. \\ E-mail: vanaya@ubiobio.cl \\ David Mora \\ GIMNAP, Departamento de Matemática, Universidad del Bío-Bío, Concepción, Chile; and Centro de \\ Investigación en Ingeniería Matemática ( $\left.\mathrm{CI}^{2} \mathrm{MA}\right)$, Universidad de Concepción, Concepción, Chile. \\ E-mail: dmora@ubiobio.cl \\ Carlos Reales \\ Departamento de Matemáticas y Estadísticas, Universidad de Córdoba, Montería, Colombia. \\ E-mail: creales@correo.unicordoba.edu.co \\ Ricardo Ruiz-Baier \\ Mathematical Institute, Oxford University, A. Wiles Building, Woodstock Road, OX2 6GG Oxford, \\ UK. E-mail: ruizbaier@maths.ox.ac.uk
}

\begin{abstract}
We introduce a new variational formulation for the Brinkman-Darcy equations formulated in terms of the scaled Brinkman vorticity and the global pressure. The velocities in each subdomain are fully decoupled through the momentum equations, and can be later recovered from the principal unknowns. A new finite element method is also proposed, consisting in equal-order Nédélec and piecewise continuous elements, for vorticity and pressure, respectively. The error analysis for the scheme is carried out in the natural norms, with bounds independent of the fluid viscosity. An adequate modification of the formulation and analysis permits us to specify the presentation to the case of axisymmetric configurations. We provide a set of numerical examples illustrating the robustness, accuracy, and efficiency of the proposed discretisation. (c) 2017 John Wiley \& Sons, Inc.
\end{abstract}

Keywords: Brinkman-Darcy coupling; vorticity-pressure formulation; finite element methods; error analysis; interface problems.

Mathematics Subject Classifications (2000): 65N30, 65N12, 35Q35, 76D07, 76S05.

\section{INTRODUCTION}

In this paper we develop the mathematical and numerical analysis for a set of partial differential equations modelling the flow of an incompressible fluid within two porous domains separated by a clearly defined interface. One medium consists of a permeable material composed by an array of low concentration fixed particles, where viscous effects of the flow are described by Brinkman equations (written in terms of vorticity, velocity and pressure). The second subdomain is a classical porous medium constituted by connected porous matrices where Darcy's law (expressed in terms of the filtration velocity and pressure) governs the non-viscous displacement of the fluid. Situations of this kind might be encountered in several applicative problems in geophysics or hydrogeology, including for instance, hydrocarbon migration, or the study of groundwater flows passing through 
soils characterised by intrinsic properties with high disparity (such as fractures). Conditions at the interface would naturally include continuity of normal velocities and additional terms accounting for normal and tangential stress preservation, whose specific form will depend on the features of the problem at hand.

If the permeability of the viscous domain goes to infinity, one readily recovers the classical Stokes flow, and the literature is populated with numerous formulations and methods to solve the StokesDarcy and Navier-Stokes-Darcy equations (see e.g. [7,9,10,12,13,15,19-21,23,26,30] and the references therein). In contrast, dedicated Brinkman-Darcy models have been studied in [11, 16, 24] (using velocity-pressure formulations), whereas the setting described above (including also the Brinkman vorticity) has been proposed only quite recently [2] (along with a fully-mixed finite element method solving for vorticity-velocity-pressure on the viscous domain and velocity-pressure on the non-viscous domain and its a posteriori error analysis developed in [3]).

Using fairly common boundary conditions (no-slip velocities on the boundary of the Brinkman domain, plus slip velocity conditions on the boundary of the Darcy domain), and exploiting regularity assumptions together with the specific form of the momentum equations on the Darcy and Brinkman domains, we are here able to decouple the velocities from the rest of the set of governing equations, in such a way that the final problem is solved only using the vorticity of the Brinkman domain and the global pressure. In fact, a similar splitting (but regarding only the Brinkman equations) has been introduced in [6] (see also related strategies in $[17,18]$ ).

The numerical method is characterised by Nédélec and piecewise continuous finite elements of degree $k \geq 1$ for the Brinkman vorticity and for the global pressure, respectively, which entails a quite low computational cost (when compared with the methods from e.g. $[2,11,16]$ ). Its optimal convergence to the corresponding weak solution is established using classical arguments and the approximation properties of the specific finite element spaces, and the obtained error bounds turn out to be fully independent of the fluid viscosity (vanishing in the Darcy limit and being relatively large in the Stokes limit). In addition, if the fluid flow and the domain at hand are considered invariant to rotations in the meridional direction, we can rewrite the problem in cylindrical coordinates, reducing the (initially three-dimensional) formulation into its axially symmetric form. Although the functional framework will necessarily undergo natural modifications, the overall structure of the analysis will remain essentially the same as in the Cartesian case.

The remainder of this paper has been organised in the following manner. The governing equations and the continuous variational formulation stated in terms of Brinkman vorticity and global pressure are presented in Section 2. Their approximation via finite elements together with the well-posedness and error analysis of the constructed schemes will be provided in Section 3. Section 4 remarks how the steps in each proof are modified in the case of axisymmetric formulations, and a few numerical tests (illustrating the convergence of our method in diverse settings) are reported in Section 5.

\section{THE MODEL PROBLEM}

Let $\Omega_{\mathrm{B}}$ and $\Omega_{\mathrm{D}}$ be open, bounded subsets of $\mathbb{R}^{d}$ (with $d \in\{2,3\}$ ) having Lipschitz-continuous boundaries, such that $\partial \Omega_{\mathrm{B}} \cap \partial \Omega_{\mathrm{D}}=\Sigma \neq \emptyset$ and $\Omega_{\mathrm{B}} \cap \Omega_{\mathrm{D}}=\emptyset$. These domains will represent the regions where viscous and non-viscous flow will be governed by Brinkman and Darcy equations, respectively. The overall porous medium is $\Omega:=\overline{\Omega_{\mathrm{B}}} \cup \overline{\Omega_{\mathrm{D}}}$ with boundary $\Gamma=\partial \Omega$. Figure 1 gives a schematic representation of the geometry in different scenarios (where the middle and right panels will be relevant in the discussion of Section 4).

Using standard notation, the system of interest can be written as the following boundary value problem. Given smooth data $\mathbf{f}_{\mathrm{B}} \in \mathrm{L}^{2}\left(\Omega_{\mathrm{B}}\right)^{d}, \mathbf{f}_{\mathrm{D}} \in \mathrm{L}^{2}\left(\Omega_{\mathrm{D}}\right)^{d}$ and $g_{\mathrm{D}} \in \mathrm{L}^{2}\left(\Omega_{\mathrm{D}}\right)$, find the velocity, scaled vorticity, and pressure in the Brinkman domain $\left(\boldsymbol{u}_{\mathrm{B}}, \boldsymbol{\omega}_{\mathrm{B}}\right.$ and $p_{\mathrm{B}}$, respectively) together with the filtration velocity and pressure in the Darcy domain $\left(\boldsymbol{u}_{\mathrm{D}}\right.$ and $\left.p_{\mathrm{D}}\right)$, such that

$$
\begin{aligned}
\kappa_{\mathrm{D}}^{-1} \boldsymbol{u}_{\mathrm{D}}+\nabla p_{\mathrm{D}} & =\mathbf{f}_{\mathrm{D}} & & \text { in } \Omega_{\mathrm{D}}, \\
\kappa_{\mathrm{B}}^{-1} \boldsymbol{u}_{\mathrm{B}}+\sqrt{\nu} \operatorname{curl} \boldsymbol{\omega}_{\mathrm{B}}+\nabla p_{\mathrm{B}} & =\mathbf{f}_{\mathrm{B}} & & \text { in } \Omega_{\mathrm{B}}, \\
\boldsymbol{\omega}_{\mathrm{B}}-\sqrt{\nu} \operatorname{curl} \boldsymbol{u}_{\mathrm{B}} & =\mathbf{0} & & \text { in } \Omega_{\mathrm{B}},
\end{aligned}
$$



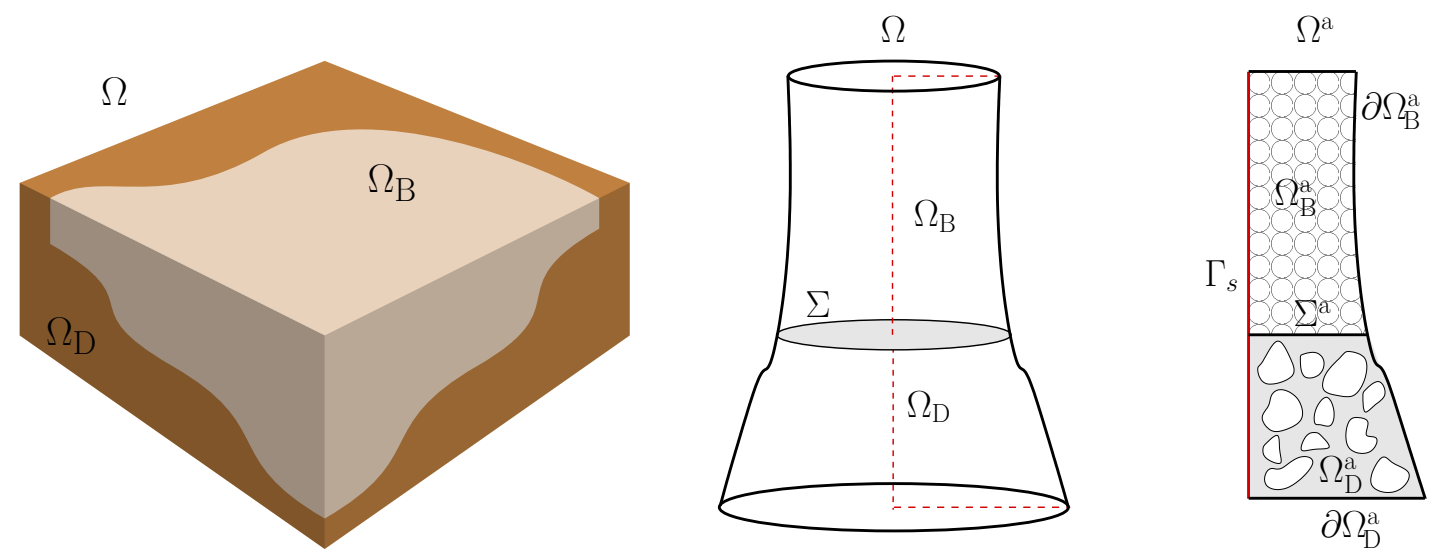

FIG. 1. Sketch of a full three dimensional domain partitioned into the Darcy and Brinkman subdomains (left), the case of an axially symmetric domain (centre), and its restriction to the meridional plane defined by the symmetry axis $\Gamma_{s}$ (right).

$$
\begin{aligned}
& \operatorname{div} \boldsymbol{u}_{\mathrm{B}}=0 \quad \text { in } \Omega_{\mathrm{D}}, \\
& \operatorname{div} \boldsymbol{u}_{\mathrm{D}}=g_{\mathrm{D}} \quad \text { in } \Omega_{\mathrm{D}}, \\
& \left(\left.\boldsymbol{u}_{\mathrm{D}}\right|_{\Omega_{\mathrm{D}}}-\left.\boldsymbol{u}_{\mathrm{B}}\right|_{\Omega_{\mathrm{B}}}\right) \cdot \boldsymbol{n}=0 \quad \text { on } \Sigma \text {, } \\
& p_{\mathrm{D}}-p_{\mathrm{B}}=0 \quad \text { on } \Sigma \text {, } \\
& \boldsymbol{\omega}_{\mathrm{B}} \times \boldsymbol{n}=\mathbf{0} \quad \text { on } \Sigma \text {, } \\
& \boldsymbol{u}_{\mathrm{D}} \cdot \boldsymbol{n}=0 \quad \text { on } \partial \Omega_{\mathrm{D}} \backslash \Sigma, \\
& \boldsymbol{u}_{\mathrm{B}}=\mathbf{0} \quad \text { on } \partial \Omega_{\mathrm{B}} \backslash \Sigma,
\end{aligned}
$$

where $\nu>0$ is the viscosity of the fluid, $\kappa_{\mathrm{D}}$ and $\kappa_{\mathrm{B}}$ are bounded, symmetric and positive definite tensors describing the permeability properties of the Darcy and Brinkman regions, respectively. Here (2.1)-(2.2) state the momentum conservation in each subdomain in the absence of inertial effects, equation (2.3) defines the constitutive relation for the additional unknown of scaled Brinkman vorticity, mass conservation is accounted for in (2.4)-(2.5), and the motion of the incompressible fluid is constrained by slip conditions on $\partial \Omega_{\mathrm{D}} \backslash \Sigma$, and no-slip conditions on $\partial \Omega_{\mathrm{B}} \backslash \Sigma$ (cf. (2.9)-(2.10)). Note that if $d=2$ then $\boldsymbol{\omega}_{\mathrm{B}}$ is the scalar vorticity, and the operator curl coincides with the two-dimensional rotated gradient.

The system is closed after providing suitable coupling conditions at the interface $\Sigma$. These conditions definitely depend on the configuration of the physical phenomenon and the formulation of the boundary value problem, but a common assumption is the continuity of normal velocities across the interface (2.6), as well as the balance of normal forces. The conservation of tangential stresses across the interface requires a much more delicate study, and we simply consider continuity of the pressure (2.7). Then, according to the simplified Beavers-Joseph-Saffman condition from [24, eq. (2.5)], for (2.7) to hold, one infers that the tangential Brinkman vorticity must vanish, as we impose in (2.8). We do refer to the motivating discussion in [29] (see also [9,22] and the references therein), and only mention that similar transmission conditions (as the ones assumed here) have been also employed in $[2,9,10,14,25]$ to produce numerical results coherent with the relevant physical scenario under consideration.

At this point we stress that an analogous set of governing equations has been introduced in [2], but prescribing both slip velocities and tangential vorticity on the whole $\partial \Omega_{\mathrm{B}}$, and therefore making use of a different functional setting leading to a variational formulation and a discretisation requiring additional unknowns, and carrying out an analysis in a substantially different manner.

In order to derive a weak formulation for (2.1)-(2.10) (on which the subsequent discretisation will be based), we recall that for any $s \geq 0$, the symbol $\|\cdot\|_{s, \Omega}$ denotes the norm of the Sobolev space $\mathrm{H}^{s}(\Omega)$ or $\mathrm{H}^{s}(\Omega)^{d}$, adopting the usual convention $\mathrm{H}^{0}(\Omega)=\mathrm{L}^{2}(\Omega)$. If $d=3$ we will also require the 
Hilbert space

$$
\mathrm{H}^{s}(\operatorname{curl} ; \Omega)=\left\{\boldsymbol{\theta} \in \mathrm{H}^{s}(\Omega)^{3}: \operatorname{curl} \boldsymbol{\theta} \in \mathrm{H}^{s}(\Omega)^{3}\right\},
$$

endowed with the norm $\|\boldsymbol{\theta}\|_{\mathrm{H}^{s}(\mathbf{c u r l} ; \Omega)}^{2}=\|\boldsymbol{\theta}\|_{s, \Omega}^{2}+\|\operatorname{curl} \boldsymbol{\theta}\|_{s, \Omega}^{2}$, and we will denote $\mathrm{H}(\mathbf{c u r l} ; \Omega)=$ $\mathrm{H}^{0}(\operatorname{curl} ; \Omega)$. If $d=2$ then we recall the characterisation $\mathrm{H}^{0}(\operatorname{curl} ; \Omega) \equiv \mathrm{H}^{1}(\Omega)$. With these considerations in mind, let us introduce the following functional spaces

$$
\mathbf{Z}:=\left\{\boldsymbol{\theta} \in \mathrm{H}\left(\operatorname{curl} ; \Omega_{\mathrm{B}}\right): \boldsymbol{\theta} \times \boldsymbol{n}=\mathbf{0} \text { on } \Sigma\right\} \quad \text { and } \quad \mathrm{Q}:=\mathrm{H}^{1}(\Omega) \cap \mathrm{L}_{0}^{2}(\Omega) .
$$

We proceed to equip $\mathrm{Q}$ with its natural norm, and $\mathbf{Z}$ with a viscosity-dependent weighted norm:

$$
\|q\|_{\mathrm{Q}}:=\left(\|q\|_{0, \Omega}^{2}+\|\nabla q\|_{0, \Omega}^{2}\right)^{1 / 2}, \quad\|\boldsymbol{\theta}\|_{\mathbf{Z}}:=\left(\|\boldsymbol{\theta}\|_{0, \Omega_{\mathrm{B}}}^{2}+\nu\|\operatorname{curl} \boldsymbol{\theta}\|_{0, \Omega_{\mathrm{B}}}^{2}\right)^{1 / 2} .
$$

If $d=2$, then $\mathbf{Z}=\left\{\boldsymbol{\theta} \in \mathrm{H}^{1}\left(\Omega_{\mathrm{B}}\right): \boldsymbol{\theta}=0\right.$ on $\left.\Sigma\right\}$.

Testing (2.3) against a generic $\boldsymbol{\theta} \in \mathbf{Z}$, integrating by parts, and using the boundary condition (2.10), we can assert that

$$
\int_{\Omega_{\mathrm{B}}} \boldsymbol{\omega}_{\mathrm{B}} \cdot \boldsymbol{\theta}-\sqrt{\nu} \int_{\Omega_{\mathrm{B}}} \boldsymbol{u}_{\mathrm{B}} \cdot \operatorname{curl} \boldsymbol{\theta}=0 \quad \forall \boldsymbol{\theta} \in \mathbf{Z} .
$$

Next, from (2.2) we readily have

$$
\kappa_{\mathrm{B}}^{-1} \boldsymbol{u}_{\mathrm{B}}=\mathbf{f}_{\mathrm{B}}-\sqrt{\nu} \operatorname{curl} \boldsymbol{\omega}_{\mathrm{B}}-\nabla p_{\mathrm{B}} \quad \text { in } \quad \Omega_{\mathrm{B}},
$$

and after replacing $(2.12)$ in $(2.11)$, we obtain

$$
\int_{\Omega_{\mathrm{B}}} \boldsymbol{\omega}_{\mathrm{B}} \cdot \boldsymbol{\theta}+\nu \int_{\Omega_{\mathrm{B}}} \kappa_{\mathrm{B}} \operatorname{curl} \boldsymbol{\omega}_{\mathrm{B}} \cdot \operatorname{curl} \boldsymbol{\theta}+\sqrt{\nu} \int_{\Omega_{\mathrm{B}}} \kappa_{\mathrm{B}} \nabla p_{\mathrm{B}} \cdot \operatorname{curl} \boldsymbol{\theta}=\sqrt{\nu} \int_{\Omega_{\mathrm{B}}} \kappa_{\mathrm{B}} \mathbf{f}_{\mathrm{B}} \cdot \operatorname{curl} \boldsymbol{\theta} \quad \forall \boldsymbol{\theta} \in \mathbf{Z} .
$$

Similarly, testing equations (2.1) and (2.2) against $\nabla q \in \mathrm{L}^{2}(\Omega)^{d}$, integrating by parts, and using the coupling conditions, we obtain

$\int_{\Omega_{\mathrm{D}}} \kappa_{\mathrm{D}} \nabla p_{\mathrm{D}} \cdot \nabla q+\sqrt{\nu} \int_{\Omega_{\mathrm{B}}} \kappa_{\mathrm{B}} \operatorname{curl} \boldsymbol{\omega}_{\mathrm{B}} \cdot \nabla q+\int_{\Omega_{\mathrm{B}}} \kappa_{\mathrm{B}} \nabla p_{\mathrm{B}} \cdot \nabla q=\int_{\Omega_{\mathrm{D}}} \kappa_{\mathrm{D}} \mathbf{f}_{\mathrm{D}} \cdot \nabla q+\int_{\Omega_{\mathrm{D}}} g_{\mathrm{D}} q+\int_{\Omega_{\mathrm{B}}} \kappa_{\mathrm{B}} \mathbf{f}_{\mathrm{B}} \cdot \nabla q$, which holds for all $q \in \mathrm{Q}$.

We can now define a global pressure field $p \in \mathrm{Q}$ such that $\left.p\right|_{\Omega_{\mathrm{B}}}=p_{\mathrm{B}}$ and $\left.p\right|_{\Omega_{\mathrm{D}}}=p_{\mathrm{D}}$, and therefore the steps above lead to the following variational formulation of (2.1)-(2.10): Find $\left(\boldsymbol{\omega}_{\mathrm{B}}, p\right) \in \mathbf{Z} \times \mathrm{Q}$ such that

$$
\mathcal{A}\left(\left(\boldsymbol{\omega}_{\mathrm{B}}, p\right),(\boldsymbol{\theta}, q)\right)=\mathcal{F}(\boldsymbol{\theta}, q) \quad \forall(\boldsymbol{\theta}, q) \in \mathbf{Z} \times \mathrm{Q},
$$

where the bilinear form $\mathcal{A}:(\mathbf{Z} \times \mathrm{Q}) \times(\mathbf{Z} \times \mathrm{Q}) \rightarrow \mathbb{R}$ and linear functional $\mathcal{F}: \mathbf{Z} \times \mathrm{Q} \rightarrow \mathbb{R}$ are defined by

$$
\begin{aligned}
\mathcal{A}\left(\left(\boldsymbol{\omega}_{\mathrm{B}}, p\right),(\boldsymbol{\theta}, q)\right) & :=\int_{\Omega_{\mathrm{B}}} \boldsymbol{\omega}_{\mathrm{B}} \cdot \boldsymbol{\theta}+\int_{\Omega_{\mathrm{B}}} \kappa_{\mathrm{B}}\left(\sqrt{\nu} \operatorname{curl} \boldsymbol{\omega}_{\mathrm{B}}+\nabla p_{\mathrm{B}}\right) \cdot(\sqrt{\nu} \operatorname{curl} \boldsymbol{\theta}+\nabla q)+\int_{\Omega_{\mathrm{D}}} \kappa_{\mathrm{D}} \nabla p_{\mathrm{D}} \cdot \nabla q, \\
\mathcal{F}(\boldsymbol{\theta}, q) & :=\int_{\Omega_{\mathrm{B}}} \kappa_{\mathrm{B}} \mathbf{f}_{\mathrm{B}} \cdot(\sqrt{\nu} \operatorname{curl} \boldsymbol{\theta}+\nabla q)+\int_{\Omega_{\mathrm{D}}} \kappa_{\mathrm{D}} \mathbf{f}_{\mathrm{D}} \cdot \nabla q+\int_{\Omega_{\mathrm{D}}} g_{\mathrm{D}} q .
\end{aligned}
$$

Should a solution to problem (2.14) exist, we note that its uniqueness is a direct consequence of the following result

Lemma 2.1. Let $\left(\boldsymbol{\omega}_{\mathrm{B}}, p\right) \in \mathbf{Z} \times \mathrm{Q}$ satisfy

$$
\mathcal{A}\left(\left(\boldsymbol{\omega}_{\mathrm{B}}, p\right),(\boldsymbol{\theta}, q)\right)=0 \quad \forall(\boldsymbol{\theta}, q) \in \mathbf{Z} \times \mathrm{Q} .
$$

Then $\left(\boldsymbol{\omega}_{\mathrm{B}}, p\right)=(\mathbf{0}, 0)$.

Proof. We take $(\boldsymbol{\theta}, q)=\left(\boldsymbol{\omega}_{\mathrm{B}}, p\right)$ as test functions, which leads to

$$
\left\|\boldsymbol{\omega}_{\mathrm{B}}\right\|_{0, \Omega_{\mathrm{B}}}^{2}+\left\|\sqrt{\nu} \operatorname{curl} \boldsymbol{\omega}_{\mathrm{B}}+\nabla p_{\mathrm{B}}\right\|_{0, \Omega_{\mathrm{B}}}^{2}+\left\|\nabla p_{\mathrm{D}}\right\|_{0, \Omega_{\mathrm{D}}}^{2}=0,
$$


implying that $\boldsymbol{\omega}_{\mathrm{B}}=\mathbf{0}$ in $\Omega_{\mathrm{B}}$ and $p=0$ in $\Omega$.

An appropriate modification of the arguments in [2] implies the existence of solutions to (2.1)-(2.10) and problem (2.14). Even if the formulation in [2] involves Lagrange multipliers accounting for the interface coupling, the regularity of the vorticity and pressure correspond to the ones we consider here. On the other hand, the well-posedness of (2.14) could be also derived adapting the steps from the very recent results in $[25$, Section 2].

\section{FINITE ELEMENT DISCRETISATION}

In this section we introduce a Galerkin scheme associated to problem (2.14), we specify the finite dimensional subspaces to employ, and analyse the well-posedness of the resulting methods using suitable assumptions on the finite element spaces. The section also contains a derivation of error estimates.

\section{Formulation and solvability}

Let $\left\{\mathcal{T}_{h}\left(\Omega_{\mathrm{B}}\right)\right\}_{h>0}$ and $\left\{\mathcal{T}_{h}\left(\Omega_{\mathrm{D}}\right)\right\}_{h>0}$ be shape-regular families of partitions of the domains $\Omega_{\mathrm{B}}$ and $\Omega_{\mathrm{D}}$, respectively, by tetrahedrons (if $d=3$, or triangles if $d=2$ ) $T$ of diameter $h_{T}$. We assume that they match in $\Sigma$ so that $\mathcal{T}_{h}\left(\Omega_{\mathrm{B}}\right) \cup \mathcal{T}_{h}\left(\Omega_{\mathrm{D}}\right)$ forms a partition of the global porous domain $\bar{\Omega}$, having meshsize $h:=\max \left\{h_{T}: T \in \mathcal{T}_{h}(\Omega)\right\}$. Given an integer $k \geq 1$ and a set $S \subset \mathbb{R}^{d}$, the space of polynomial functions defined in $S$ and having total degree $\leq k$ will be denoted by $\mathcal{P}_{k}(S)$.

For any $T \in \mathcal{T}_{h}\left(\Omega_{\mathrm{B}}\right)$ we recall the definition of the local Nédélec space:

$$
\mathbb{N}_{k}(T):=\mathcal{P}_{k-1}(T)^{3} \oplus R_{k}(T),
$$

where $R_{k}(T)$ is the subspace of $\mathcal{P}_{k}(T)^{3}$ composed by homogeneous polynomials of degree $k$, and orthogonal to $\boldsymbol{x}$. The finite element spaces for the approximation of the Brinkman vorticity and the global pressure are then defined as

$$
\begin{aligned}
& \mathbf{Z}_{h}:=\left\{\boldsymbol{\theta}_{h} \in \mathbf{Z}:\left.\boldsymbol{\theta}_{h}\right|_{T} \in \mathbb{N}_{k}(T) \quad \forall T \in \mathcal{T}_{h}\left(\Omega_{\mathrm{B}}\right)\right\}, \\
& \mathrm{Q}_{h}:=\left\{q_{h} \in \mathrm{Q}:\left.q_{h}\right|_{T} \in \mathcal{P}_{k}(T) \quad \forall T \in \mathcal{T}_{h}(\Omega)\right\},
\end{aligned}
$$

which are subspaces of $\mathbf{Z}$ and $\mathrm{Q}$, respectively. If $d=2$, then $\mathbf{Z}_{h}=\left\{\boldsymbol{\theta}_{h} \in \mathbf{Z}:\left.\boldsymbol{\theta}_{h}\right|_{T} \in \mathcal{P}_{k}(T) \quad \forall T \in\right.$ $\left.\mathcal{T}_{h}\left(\Omega_{\mathrm{B}}\right)\right\}$.

Therefore, a Galerkin scheme associated with the continuous variational formulation (2.14) reads as follows: Find $\left(\boldsymbol{\omega}_{\mathrm{B}, h}, p_{h}\right) \in \mathbf{Z}_{h} \times \mathrm{Q}_{h}$ such that

$$
\mathcal{A}\left(\left(\boldsymbol{\omega}_{\mathrm{B}, h}, p_{h}\right),\left(\boldsymbol{\theta}_{h}, q_{h}\right)\right)=\mathcal{F}\left(\boldsymbol{\theta}_{h}, q_{h}\right) \quad \forall\left(\boldsymbol{\theta}_{h}, q_{h}\right) \in \mathbf{Z}_{h} \times \mathrm{Q}_{h} .
$$

Theorem 3.1 (Solvability of the Galerkin method). The discrete problem (3.3) is well-posed.

Proof. Since (3.3) consists of a square linear system, it suffices to establish the uniqueness. Assuming that the data are homogeneous $\mathbf{f}_{\mathrm{B}}=\mathbf{0}, \mathbf{f}_{\mathrm{D}}=\mathbf{0}$ and $g_{\mathrm{D}}=0$, we can choose $\left(\boldsymbol{\theta}_{h}, q_{h}\right)=$ $\left(\boldsymbol{\omega}_{\mathrm{B}, h}, p_{h}\right)$ as test functions in the Galerkin formulation. This leads to

$$
\left\|\boldsymbol{\omega}_{\mathrm{B}, h}\right\|_{0, \Omega_{\mathrm{B}}}^{2}+\left\|\sqrt{\nu} \operatorname{curl} \boldsymbol{\omega}_{\mathrm{B}, h}+\nabla p_{\mathrm{B}, h}\right\|_{0, \Omega_{\mathrm{B}}}^{2}+\left\|\nabla p_{\mathrm{D}, h}\right\|_{0, \Omega_{\mathrm{D}}}^{2}=0,
$$

implying that $\boldsymbol{\omega}_{\mathrm{B}, h}=\mathbf{0}$ in $\Omega_{\mathrm{B}}$ and $p_{h}=0$ in $\Omega$.

\section{Error estimates}

Let us introduce, for $s>1 / 2$, the Nédélec global interpolation operator $\mathcal{R}_{h}: \mathrm{H}^{s}\left(\operatorname{curl} ; \Omega_{\mathrm{B}}\right) \cap \mathbf{Z} \rightarrow \mathbf{Z}_{h}$ (cf. [1]), satisfying the following approximation property.

Lemma 3.2. For all $\boldsymbol{\theta}_{\mathrm{B}} \in \mathrm{H}^{s}\left(\mathbf{c u r l} ; \Omega_{\mathrm{B}}\right)$ with $s \in(1 / 2, k]$, there exists $C>0$ independent of $h$, such that

$$
\left\|\boldsymbol{\theta}_{\mathrm{B}}-\mathcal{R}_{h} \boldsymbol{\theta}_{\mathrm{B}}\right\|_{\mathbf{Z}} \leq C h^{s}\left\|\boldsymbol{\theta}_{\mathrm{B}}\right\|_{\mathrm{H}^{s}\left(\operatorname{curl} ; \Omega_{\mathrm{B}}\right)} .
$$


On the other hand, for all $s>1 / 2$, the usual Lagrange interpolant $\Pi_{h}: \mathrm{H}^{1+s}(\Omega) \cap \mathrm{Q} \rightarrow \mathrm{Q}_{h}$ features a similar property.

Lemma 3.3. For all $q \in \mathrm{H}^{1+s}(\Omega), s \in(1 / 2, k]$ there exists $C>0$, independent of $h$, such that

$$
\left\|q-\Pi_{h} q\right\|_{\mathrm{Q}} \leq C h^{s}\|q\|_{\mathrm{H}^{1+s}(\Omega)} .
$$

The following auxiliary result will be used in the derivation of the error estimates.

Lemma 3.4. For all $\left(\boldsymbol{\theta}_{\mathrm{B}}, q\right) \in \mathbf{Z} \times \mathrm{Q}$, there exist $C_{1}, C_{2}>0$ such that

$$
C_{1}\left(\left\|\boldsymbol{\theta}_{\mathrm{B}}\right\|_{0, \Omega_{\mathrm{B}}}^{2}+\left\|\sqrt{\nu} \operatorname{curl} \boldsymbol{\theta}_{\mathrm{B}}+\nabla q_{\mathrm{B}}\right\|_{0, \Omega_{\mathrm{B}}}^{2}+\left\|\nabla q_{\mathrm{D}}\right\|_{0, \Omega_{\mathrm{D}}}^{2}+\|q\|_{0, \Omega}^{2}\right) \leq \mathcal{A}\left(\left(\boldsymbol{\theta}_{\mathrm{B}}, q\right),\left(\boldsymbol{\theta}_{\mathrm{B}}, q\right)\right),
$$

and

$$
\mathcal{A}\left(\left(\boldsymbol{\theta}_{\mathrm{B}}, q\right),\left(\boldsymbol{\theta}_{\mathrm{B}}, q\right)\right) \leq C_{2}\left(\left\|\boldsymbol{\theta}_{\mathrm{B}}\right\|_{0, \Omega_{\mathrm{B}}}^{2}+\left\|\sqrt{\nu} \operatorname{curl} \boldsymbol{\theta}_{\mathrm{B}}+\nabla q_{\mathrm{B}}\right\|_{0, \Omega_{\mathrm{B}}}^{2}+\left\|\nabla q_{\mathrm{D}}\right\|_{0, \Omega_{\mathrm{D}}}^{2}+\|q\|_{0, \Omega}^{2}\right) .
$$

Proof. For a given $\left(\boldsymbol{\theta}_{\mathrm{B}}, q\right) \in \mathbf{Z} \times \mathrm{Q}$ there holds that

$$
\begin{aligned}
\|q\|_{0, \Omega} & \leq\|\nabla q\|_{-1, \Omega}:=\sup _{\boldsymbol{v} \in \mathrm{H}_{0}^{1}(\Omega)^{d} \backslash\{\mathbf{0}\}} \frac{(\boldsymbol{v}, \nabla q)_{0, \Omega}}{\|\boldsymbol{v}\|_{1, \Omega}}=\sup _{\boldsymbol{v} \in \mathrm{H}_{0}^{1}(\Omega)^{d} \backslash\{\mathbf{0}\}} \frac{\left(\boldsymbol{v}, \nabla q_{\mathrm{B}}\right)_{0, \Omega_{\mathrm{B}}}+\left(\boldsymbol{v}, \nabla q_{\mathrm{D}}\right)_{0, \Omega_{\mathrm{D}}}}{\|\boldsymbol{v}\|_{1, \Omega}} \\
& =\sup _{\boldsymbol{v} \in \mathrm{H}_{0}^{1}(\Omega)^{d} \backslash\{\mathbf{0}\}} \frac{\left(\boldsymbol{v}, \nabla q_{\mathrm{B}}+\sqrt{\nu} \operatorname{curl} \boldsymbol{\theta}_{\mathrm{B}}\right)_{0, \Omega_{\mathrm{B}}}-\left(\boldsymbol{v}, \sqrt{\nu} \operatorname{curl} \boldsymbol{\theta}_{\mathrm{B}}\right)_{0, \Omega_{\mathrm{B}}}+\left(\boldsymbol{v}, \nabla q_{\mathrm{D}}\right)_{0, \Omega_{\mathrm{D}}}}{\|\boldsymbol{v}\|_{1, \Omega}} \\
& =\sup _{\boldsymbol{v} \in \mathrm{H}_{0}^{1}(\Omega)^{d} \backslash\{\mathbf{0}\}} \frac{\left(\boldsymbol{v}, \nabla q_{\mathrm{B}}+\sqrt{\nu} \operatorname{curl} \boldsymbol{\theta}_{\mathrm{B}}\right)_{0, \Omega_{\mathrm{B}}}-\sqrt{\nu}\left(\boldsymbol{\theta}_{\mathrm{B}}, \operatorname{curl} \boldsymbol{v}\right)_{0, \Omega_{\mathrm{B}}}+\left(\boldsymbol{v}, \nabla q_{\mathrm{D}}\right)_{0, \Omega_{\mathrm{D}}}}{\|\boldsymbol{v}\|_{1, \Omega}} \\
& \leq \sup _{\boldsymbol{v} \in \mathrm{H}_{0}^{1}(\Omega)^{d} \backslash\{\mathbf{0}\}} \frac{\left(\left\|\nabla q_{\mathrm{B}}+\sqrt{\nu} \operatorname{curl} \boldsymbol{\theta}_{\mathrm{B}}\right\|_{0, \Omega_{\mathrm{B}}}+\left\|\nabla q_{\mathrm{D}}\right\|_{0, \Omega_{\mathrm{D}}}\right)\|\boldsymbol{v}\|_{0, \Omega}+\sqrt{\nu}\left\|\boldsymbol{\theta}_{\mathrm{B}}\right\|_{0, \Omega_{\mathrm{B}}}\|\operatorname{curl} \boldsymbol{v}\|_{0, \Omega_{\mathrm{B}}}}{\|\boldsymbol{v}\|_{1, \Omega}} \\
& \leq C\left(\left\|\nabla q_{\mathrm{B}}+\sqrt{\nu} \operatorname{curl} \boldsymbol{\theta}_{\mathrm{B}}\right\|_{0, \Omega_{\mathrm{B}}}+\left\|\boldsymbol{\theta}_{\mathrm{B}}\right\|_{0, \Omega_{\mathrm{B}}}+\left\|\nabla q_{\mathrm{D}}\right\|_{0, \Omega_{\mathrm{D}}}\right) .
\end{aligned}
$$

Thus (3.4) follows. Finally, (3.5) is obtained directly from triangle inequality.

Theorem 3.5 (Optimal convergence). Assume that $\omega_{\mathrm{B}} \in \mathrm{H}^{s}\left(\operatorname{curl} ; \Omega_{\mathrm{B}}\right)$, and $p \in \mathrm{H}^{1+s}(\Omega)$, for some $s \in(1 / 2, k]$. Then, there exists $C>0$ independent of $h$ and $\nu$, such that

$$
\begin{aligned}
\left\|\boldsymbol{\omega}_{\mathrm{B}}-\boldsymbol{\omega}_{\mathrm{B}, h}\right\|_{0, \Omega_{\mathrm{B}}}+\left\|\sqrt{\nu} \operatorname{curl}\left(\boldsymbol{\omega}_{\mathrm{B}}-\boldsymbol{\omega}_{\mathrm{B}, h}\right)+\nabla\left(p_{\mathrm{B}}-p_{\mathrm{B}, h}\right)\right\|_{0, \Omega_{\mathrm{B}}} \\
\quad+\left\|\nabla\left(p_{\mathrm{D}}-p_{\mathrm{D}, h}\right)\right\|_{0, \Omega_{\mathrm{D}}}+\left\|p-p_{h}\right\|_{0, \Omega} \leq C h^{s}\left(\left\|\boldsymbol{\omega}_{\mathrm{B}}\right\|_{\mathrm{H}^{s}\left(\operatorname{curl} ; \Omega_{\mathrm{B}}\right)}+\|p\|_{\mathrm{H}^{1+s}(\Omega)}\right) .
\end{aligned}
$$

Proof. The result is a consequence of the error equation

$$
\mathcal{A}\left(\left(\boldsymbol{\omega}_{\mathrm{B}}-\boldsymbol{\omega}_{\mathrm{B}, h}, p-p_{h}\right),\left(\boldsymbol{\theta}_{h}, q_{h}\right)\right)=0 \quad \forall\left(\boldsymbol{\theta}_{h}, q_{h}\right) \in \mathbf{Z}_{h} \times \mathrm{Q}_{h},
$$

in combination with Lemmas 3.4, 3.2 and 3.3.

Our next result establishes a duality argument, permitting us to improve the convergence of the vorticity and global pressure errors in the $L^{2}$-norm.

Theorem 3.6 (An $L^{2}$-estimate). Assume that $\boldsymbol{\omega}_{\mathrm{B}} \in \mathrm{H}^{s}\left(\operatorname{curl} ; \Omega_{\mathrm{B}}\right)$, and $p \in \mathrm{H}^{1+s}(\Omega)$, for some $s \in(1 / 2, k]$. Then, there exists $C>0$ independent of $h$ and $\nu$, such that

$$
\left\|\boldsymbol{\omega}_{\mathrm{B}}-\boldsymbol{\omega}_{\mathrm{B}, h}\right\|_{0, \Omega_{\mathrm{B}}}+\left\|p-p_{h}\right\|_{0, \Omega} \leq C h^{1+s}\left(\left\|\boldsymbol{\omega}_{\mathrm{B}}\right\|_{\mathrm{H}^{s}\left(\mathbf{c u r l} ; \Omega_{\mathrm{B}}\right)}+\|p\|_{\mathrm{H}^{1+s}(\Omega)}\right) .
$$

Proof. Let us consider the following well-posed problem

$$
\mathcal{A}\left((\widetilde{\boldsymbol{\omega}}, \tilde{p}),\left(\boldsymbol{\theta}_{\mathrm{B}}, q\right)\right)=\int_{\Omega_{\mathrm{B}}}\left(\boldsymbol{\omega}_{\mathrm{B}}-\boldsymbol{\omega}_{\mathrm{B}, h}\right) \cdot \boldsymbol{\theta}_{\mathrm{B}}+\int_{\Omega}\left(p-p_{h}\right) q \quad \forall\left(\boldsymbol{\theta}_{\mathrm{B}}, q\right) \in \mathbf{Z} \times \mathrm{Q},
$$

and let us assume the additional regularity $\widetilde{\boldsymbol{\omega}} \in \mathrm{H}^{1}\left(\operatorname{curl} ; \Omega_{\mathrm{B}}\right)$ and $\tilde{p} \in \mathrm{H}^{2}(\Omega)$. Moreover, we also assume that there exists a constant $C>0$, independent of $\nu$ such that

$$
\|\widetilde{\boldsymbol{\omega}}\|_{\mathrm{H}^{1}\left(\mathbf{c u r l} ; \Omega_{\mathrm{B}}\right)}+\|\tilde{p}\|_{\mathrm{H}^{2}(\Omega)} \leq C\left(\left\|\boldsymbol{\omega}_{\mathrm{B}}-\boldsymbol{\omega}_{\mathrm{B}, h}\right\|_{0, \Omega_{\mathrm{B}}}+\left\|p-p_{h}\right\|_{0, \Omega}\right) .
$$


Then we can choose $\left(\boldsymbol{\theta}_{\mathrm{B}}, q\right)=\left(\boldsymbol{\omega}_{\mathrm{B}}-\boldsymbol{\omega}_{\mathrm{B}, h}, p-p_{h}\right)$ in $(3.7)$, to obtain

$$
\left\|\boldsymbol{\omega}_{\mathrm{B}}-\boldsymbol{\omega}_{\mathrm{B}, h}\right\|_{0, \Omega_{\mathrm{B}}}^{2}+\left\|p-p_{h}\right\|_{0, \Omega}^{2}=\mathcal{A}\left((\widetilde{\boldsymbol{\omega}}, \tilde{p}),\left(\boldsymbol{\omega}_{\mathrm{B}}-\boldsymbol{\omega}_{\mathrm{B}, h}, p-p_{h}\right)\right),
$$

and the required result follows as a consequence of (3.6) together with Lemmas 3.2, 3.3, the bound (3.8), and Theorem 3.5.

\section{Recovering the velocity field}

The solution of the continuous and discrete problems (2.14) and (3.3), deliver the Brinkman vorticity and global pressure $\left(\boldsymbol{\omega}_{\mathrm{B}}, p\right) \in \mathbf{Z} \times \mathrm{Q}$ and $\left(\boldsymbol{\omega}_{\mathrm{B}, h}, p_{h}\right) \in \mathbf{Z}_{h} \times \mathrm{Q}_{h}$, respectively. From these quantities we can readily obtain the continuous and discrete velocities. In fact, according to (2.12) and (2.1) we have

$$
\begin{array}{ll}
\boldsymbol{u}_{\mathrm{B}}=\kappa_{\mathrm{B}}\left(\mathbf{f}_{\mathrm{B}}-\sqrt{\nu} \operatorname{curl} \boldsymbol{\omega}_{\mathrm{B}}-\nabla p_{\mathrm{B}}\right) & \text { in } \Omega_{\mathrm{B}}, \\
\boldsymbol{u}_{\mathrm{D}}=\kappa_{\mathrm{D}}\left(\mathbf{f}_{\mathrm{D}}-\nabla p_{\mathrm{D}}\right) & \text { in } \Omega_{\mathrm{D}} .
\end{array}
$$

Similarly, at the discrete level (3.9)-(3.10) correspond to computing the Brinkman and Darcy velocities as a post-process from the discrete Brinkman vorticity and the global pressure:

$$
\begin{array}{ll}
\boldsymbol{u}_{\mathrm{B}, h}=\kappa_{\mathrm{B}}\left(\mathcal{P}_{h} \mathbf{f}_{\mathrm{B}}-\sqrt{\nu} \operatorname{curl} \boldsymbol{\omega}_{\mathrm{B}, h}-\nabla p_{\mathrm{B}, h}\right) & \text { in } \Omega_{\mathrm{B}}, \\
\boldsymbol{u}_{\mathrm{D}, h}=\kappa_{\mathrm{D}}\left(\mathcal{P}_{h} \mathbf{f}_{\mathrm{D}}-\nabla p_{\mathrm{D}, h}\right) & \text { in } \Omega_{\mathrm{D}},
\end{array}
$$

where $\mathcal{P}_{h}: \mathrm{L}^{2}(\Omega)^{d} \rightarrow U_{h}:=\left\{\boldsymbol{v}_{h} \in \mathrm{L}^{2}(\Omega)^{d}:\left.\boldsymbol{v}_{h}\right|_{T} \in \mathcal{P}_{k-1}(T)^{d} \quad \forall T \in \mathcal{T}_{h}(\Omega)\right\}$ is the $\mathrm{L}^{2}$-orthogonal projector satisfying for any $s \in(0, k]$

$$
\left\|\boldsymbol{v}-\mathcal{P}_{h} \boldsymbol{v}\right\|_{0, \Omega} \leq C h^{s}\|\boldsymbol{v}\|_{s, \Omega}
$$

Note that both $\boldsymbol{u}_{\mathrm{B}, h}$ and $\boldsymbol{u}_{\mathrm{D}, h}$ are element-wise discontinuous, and, should further features be sought (e.g. local divergence-free), one requires additional projection steps (see for instance [27]). In any case, the rate of convergence of the velocity postprocessing can be quantified as follows.

Theorem 3.7 Convergence of the velocity postprocessing. Let $\boldsymbol{\omega}_{\mathrm{B}} \in \mathbf{Z}$ and $p \in \mathrm{Q}$ be the unique solutions of (2.14) and $\boldsymbol{\omega}_{\mathrm{B}, h} \in \mathbf{Z}_{h}$ and $p_{h} \in \mathrm{Q}_{h}$ be the unique solutions of (3.3). Assume that $\boldsymbol{\omega}_{\mathrm{B}} \in \mathrm{H}^{s}\left(\mathbf{c u r l} ; \Omega_{\mathrm{B}}\right), p \in \mathrm{H}^{1+s}(\Omega), \mathbf{f}_{\mathrm{D}} \in \mathrm{H}^{s}\left(\Omega_{\mathrm{D}}\right)^{d}$ and $\mathbf{f}_{\mathrm{B}} \in \mathrm{H}^{s}\left(\Omega_{\mathrm{B}}\right)^{d}$, for some $s \in(1 / 2, k]$. Then, there exists $C>0$ independent of $h$ and $\nu$, such that

$$
\left\|\boldsymbol{u}_{\mathrm{B}}-\boldsymbol{u}_{\mathrm{B}, h}\right\|_{0, \Omega_{\mathrm{B}}}+\left\|\boldsymbol{u}_{\mathrm{D}}-\boldsymbol{u}_{\mathrm{D}, h}\right\|_{0, \Omega_{\mathrm{D}}} \leq C h^{s}\left(\left\|\mathbf{f}_{\mathrm{B}}\right\|_{s, \Omega_{\mathrm{B}}}+\left\|\mathbf{f}_{\mathrm{D}}\right\|_{s, \Omega_{\mathrm{D}}}+\left\|\boldsymbol{\omega}_{\mathrm{B}}\right\|_{\mathrm{H}^{s}\left(\mathbf{c u r l} ; \Omega_{\mathrm{B}}\right)}+\|p\|_{1+s, \Omega}\right) .
$$

Proof. Collecting the results from (3.9), (3.10), (3.11) and (3.12), and using triangle inequality, it follows that

$$
\begin{aligned}
\left\|\boldsymbol{u}_{\mathrm{B}}-\boldsymbol{u}_{\mathrm{B}, h}\right\|_{0, \Omega_{\mathrm{B}}}+\left\|\boldsymbol{u}_{\mathrm{D}}-\boldsymbol{u}_{\mathrm{D}, h}\right\|_{0, \Omega_{\mathrm{D}}} \leq & C\left(\left\|\mathbf{f}_{\mathrm{B}}-\mathcal{P}_{h} \mathbf{f}_{\mathrm{B}}\right\|_{0, \Omega_{\mathrm{B}}}+\left\|\mathbf{f}_{\mathrm{D}}-\mathcal{P}_{h} \mathbf{f}_{\mathrm{D}}\right\|_{0, \Omega_{\mathrm{D}}}\right. \\
& +\left\|\sqrt{\nu} \operatorname{curl}\left(\boldsymbol{\omega}_{\mathrm{B}}-\boldsymbol{\omega}_{\mathrm{B}, h}\right)+\nabla\left(p_{\mathrm{B}}-p_{\mathrm{B}, h}\right)\right\|_{0, \Omega_{\mathrm{B}}} \\
& \left.+\left\|\nabla\left(p_{\mathrm{D}}-p_{\mathrm{D}, h}\right)\right\|_{0, \Omega_{\mathrm{D}}}\right) .
\end{aligned}
$$

Then the desired result is obtained from Theorem 3.5 and (3.13).

\section{REDUCTION TO THE AXISYMMETRIC CASE}

Let us now consider $d=3$ and assume that the data, the porous domain $\Omega$ along with its subdomains, and the expected flow properties are all symmetric with respect to a given axis $\Gamma_{s}$. Therefore the governing equations can be redefined in the meridional domain $\Omega^{\mathrm{a}}$ (see Figure 1, right panel), the Brinkman and Darcy velocities involve only the radial and vertical components, and the Brinkman vorticity is now the scalar field $\omega_{\mathrm{B}}=\sqrt{\nu} \operatorname{rot} \boldsymbol{u}_{\mathrm{B}}{ }^{1}$. In analogy to $(2.1)-(2.10)$, the boundary value

\footnotetext{
${ }^{1}$ We recall that the needed differential operators in axisymmetric coordinates are $\operatorname{div}_{\mathrm{a}} \boldsymbol{v}:=\partial_{z} v_{z}+\frac{1}{r} \partial_{r}\left(r v_{r}\right)$, $\operatorname{rot} \boldsymbol{v}:=\partial_{r} v_{z}-\partial_{z} v_{r}, \nabla \varphi:=\left(\partial_{r} \varphi, \partial_{z} \varphi\right)^{T}$, and $\operatorname{curl}_{\mathbf{a}} \varphi:=\left(\partial_{z} \varphi,-r^{-1} \partial_{r}(r \varphi)\right)^{T}$.
} 
problem now reads

$$
\begin{aligned}
& \kappa_{\mathrm{D}}^{-1} \boldsymbol{u}_{\mathrm{D}}+\nabla p_{\mathrm{D}}=\mathbf{f}_{\mathrm{D}}, \quad \text { and } \quad \operatorname{div}_{\mathrm{a}} \boldsymbol{u}_{\mathrm{D}}=g_{\mathrm{D}} \quad \text { in } \Omega_{\mathrm{D}}^{\mathrm{a}}, \\
& \kappa_{\mathrm{B}}^{-1} \boldsymbol{u}_{\mathrm{B}}+\sqrt{\nu} \operatorname{curl}_{\mathrm{a}} \omega_{\mathrm{B}}+\nabla p_{\mathrm{B}}=\mathbf{f}_{\mathrm{B}}, \operatorname{div}_{\mathrm{a}} \boldsymbol{u}_{\mathrm{B}}=0, \quad \text { and } \omega_{\mathrm{B}}-\sqrt{\nu} \operatorname{rot} \boldsymbol{u}_{\mathrm{B}}=0 \quad \text { in } \Omega_{\mathrm{B}}^{\mathrm{a}}, \\
& \left(\left.\boldsymbol{u}_{\mathrm{B}}\right|_{\Omega_{\mathrm{B}}^{\mathrm{a}}}-\left.\boldsymbol{u}_{\mathrm{D}}\right|_{\Omega_{\mathrm{D}}^{\mathrm{a}}}\right) \cdot \boldsymbol{n}=0,\left.\quad p_{\mathrm{B}}\right|_{\Omega_{\mathrm{B}}^{\mathrm{a}}}-\left.p_{\mathrm{D}}\right|_{\Omega_{\mathrm{D}}^{\mathrm{a}}}=0, \quad \text { and } \quad \omega_{\mathrm{B}}=0 \quad \text { on } \Sigma^{\mathrm{a}} \text {, } \\
& \boldsymbol{u}_{\mathrm{D}} \cdot \boldsymbol{n}=0 \quad \text { on } \partial \Omega_{\mathrm{D}}^{\mathrm{a}} \backslash \Sigma^{\mathrm{a}}, \\
& \boldsymbol{u}_{\mathrm{B}}=\mathbf{0} \quad \text { on } \partial \Omega_{\mathrm{B}}^{\mathrm{a}} \backslash \Sigma^{\mathrm{a}} \text {. }
\end{aligned}
$$

In order to adapt the analysis presented in the previous sections to axisymmetric enclosures, we require a modification of the functional spaces as advanced in [28]. We begin by denoting $\mathrm{L}_{\alpha}^{p}\left(\Omega^{\mathrm{a}}\right)$ the weighted Lebesgue space of measurable functions $\varphi$ satisfying

$$
\|\varphi\|_{\mathrm{L}_{\alpha}^{p}\left(\Omega^{\mathrm{a}}\right)}^{p}:=\int_{\Omega^{\mathrm{a}}}|\varphi|^{p} r^{\alpha} \mathrm{d} r \mathrm{~d} z<\infty,
$$

and $\mathrm{L}_{1,0}^{2}\left(\Omega^{\mathrm{a}}\right)$ will denote its restriction to functions with zero weighted integral. The weighted Sobolev space $\mathrm{H}_{r}^{k}\left(\Omega^{\mathrm{a}}\right)$ consists of all functions in $\mathrm{L}_{1}^{2}\left(\Omega^{\mathrm{a}}\right)$ whose derivatives of order $\leq k$ are also in $\mathrm{L}_{1}^{2}\left(\Omega^{\mathrm{a}}\right)$, and its semi-norm is defined as usual. For $k=1$ we have

$$
|\varphi|_{\mathrm{H}_{1}^{1}\left(\Omega^{\mathrm{a}}\right)}^{2}:=\int_{\Omega^{\mathrm{a}}}\left(\left|\partial_{r} \varphi\right|^{2}+\left|\partial_{z} \varphi\right|^{2}\right) r \mathrm{~d} r \mathrm{~d} z,
$$

and the space $\widetilde{\mathrm{H}}_{1}^{1}\left(\Omega_{\mathrm{B}}^{\mathrm{a}}\right):=\mathrm{H}_{1}^{1}\left(\Omega_{\mathrm{B}}^{\mathrm{a}}\right) \cap \mathrm{L}_{-1}^{2}\left(\Omega_{\mathrm{B}}^{\mathrm{a}}\right)$, equipped with the norm

$$
\|\varphi\|_{\widetilde{\mathrm{H}}_{1}^{1}\left(\Omega_{\mathrm{B}}^{\mathrm{a}}\right)}:=\left(\|\varphi\|_{\mathrm{L}_{1}^{2}\left(\Omega_{\mathrm{B}}^{\mathrm{a}}\right)}^{2}+\nu|\varphi|_{\mathrm{H}_{1}^{1}\left(\Omega_{\mathrm{B}}^{\mathrm{a}}\right)}^{2}+\nu\|\varphi\|_{\mathrm{L}_{-1}^{2}\left(\Omega_{\mathrm{B}}^{\mathrm{a}}\right)}^{2}\right)^{1 / 2},
$$

is a Hilbert space. The space $\mathrm{H}\left(\operatorname{curl}_{\mathrm{a}}, \Omega_{\mathrm{B}}^{\mathrm{a}}\right):=\left\{\varphi \in \mathrm{L}_{1}^{2}\left(\Omega_{\mathrm{B}}^{\mathrm{a}}\right): \operatorname{curl}_{\mathrm{a}} \varphi \in \mathrm{L}_{1}^{2}\left(\Omega_{\mathrm{B}}^{\mathrm{a}}\right)\right\}$ will be provided with the norm $\|\varphi\|_{\mathrm{H}\left(\operatorname{curl}_{\mathbf{a}}, \Omega_{\mathrm{B}}^{\mathrm{a}}\right)}^{2}=\|\varphi\|_{\mathrm{L}_{1}^{2}\left(\Omega_{\mathrm{B}}^{\mathrm{a}}\right)}^{2}+\nu\left\|\operatorname{curl}_{\mathrm{a}} \varphi\right\|_{\mathrm{L}_{1}^{2}\left(\Omega_{\mathrm{B}}^{\mathrm{a}}\right)^{2}}^{2}$, and we notice that $\|\cdot\|_{\mathrm{H}\left(\operatorname{curl}_{\mathbf{a}}, \Omega_{\mathrm{B}}^{\mathrm{a}}\right)}$ and $\|\cdot\|_{\widetilde{\mathrm{H}}_{1}^{1}\left(\Omega_{\mathrm{B}}^{\mathrm{a}}\right)}$ are equivalent norms.

A variational formulation for system (4.1)-(4.5) can be derived as in Section 2. In particular, we repeat the arguments in (2.11)-(2.13) together with Lemmas 1.2 and 1.3 from [5], to obtain the following variational formulation: Find $\left(\omega_{\mathrm{B}}, p\right) \in \mathbf{Z}^{\mathrm{a}} \times \mathrm{Q}^{\mathrm{a}}$ such that

$$
\mathcal{A}^{\mathrm{a}}\left(\left(\omega_{\mathrm{B}}, p\right),(\theta, q)\right)=\mathcal{F}^{\mathrm{a}}(\theta, q) \quad \forall(\theta, q) \in \mathbf{Z}^{\mathrm{a}} \times \mathrm{Q}^{\mathrm{a}},
$$

where the associated functional spaces are

$$
\mathbf{Z}^{\mathrm{a}}:=\left\{\varphi \in \widetilde{\mathrm{H}}_{1}^{1}\left(\Omega_{\mathrm{B}}^{\mathrm{a}}\right): \varphi=0 \text { on } \Sigma^{\mathrm{a}}\right\}, \quad \mathrm{Q}^{\mathrm{a}}:=\mathrm{H}_{1}^{1}\left(\Omega^{\mathrm{a}}\right) \cap \mathrm{L}_{1,0}^{2}\left(\Omega^{\mathrm{a}}\right),
$$

and the bilinear form $\mathcal{A}^{\mathrm{a}}:\left(\mathbf{Z}^{\mathrm{a}} \times \mathrm{Q}^{\mathrm{a}}\right) \times\left(\mathbf{Z}^{\mathrm{a}} \times \mathrm{Q}^{\mathrm{a}}\right) \rightarrow \mathbb{R}$ and linear functional $\mathcal{F}^{\mathrm{a}}: \mathbf{Z}^{\mathrm{a}} \times \mathrm{Q}^{\mathrm{a}} \rightarrow \mathbb{R}$ are now specified as

$$
\begin{aligned}
\mathcal{A}^{\mathrm{a}}\left(\left(\omega_{\mathrm{B}}, p\right),(\theta, q)\right):= & \int_{\Omega_{\mathrm{B}}^{\mathrm{a}}} \omega_{\mathrm{B}} \theta r \mathrm{~d} r \mathrm{~d} z+\int_{\Omega_{\mathrm{B}}^{\mathrm{a}}} \kappa_{\mathrm{B}}\left(\sqrt{\nu} \operatorname{curl}_{\mathrm{a}} \omega_{\mathrm{B}}+\nabla p_{\mathrm{B}}\right) \cdot\left(\sqrt{\nu} \operatorname{curl}_{\mathrm{a}} \theta+\nabla q\right) r \mathrm{~d} r \mathrm{~d} z \\
& +\int_{\Omega_{\mathrm{D}}^{\mathrm{a}}} \kappa_{\mathrm{D}} \nabla p_{\mathrm{D}} \cdot \nabla q r \mathrm{~d} r \mathrm{~d} z, \\
\mathcal{F}^{\mathrm{a}}(\theta, q):= & \int_{\Omega_{\mathrm{B}}^{\mathrm{a}}} \kappa_{\mathrm{B}} \mathbf{f}_{\mathrm{B}} \cdot\left(\sqrt{\nu} \operatorname{curl}_{\mathbf{a}} \theta+\nabla q\right) r \mathrm{~d} r \mathrm{~d} z+\int_{\Omega_{\mathrm{D}}^{\mathrm{a}}} \kappa_{\mathrm{D}} \mathbf{f}_{\mathrm{D}} \cdot \nabla q r \mathrm{~d} r \mathrm{~d} z+\int_{\Omega_{\mathrm{D}}^{\mathrm{a}}} g_{\mathrm{D}} q r \mathrm{~d} r \mathrm{~d} z,
\end{aligned}
$$

Introducing the finite element subspaces (for any $k \geq 1$ )

$$
\begin{aligned}
& \mathbf{Z}_{h}^{\mathrm{a}}:=\left\{\theta_{h} \in \mathbf{Z}^{\mathrm{a}}:\left.\theta_{h}\right|_{T} \in \mathcal{P}_{k}(T) \forall T \in \mathcal{T}_{h}\left(\Omega_{\mathrm{B}}^{\mathrm{a}}\right)\right\}, \\
& \mathrm{Q}_{h}^{\mathrm{a}}:=\left\{q_{h} \in \mathrm{Q}^{\mathrm{a}}:\left.q_{h}\right|_{T} \in \mathcal{P}_{k}(T) \forall T \in \mathcal{T}_{h}\left(\Omega^{\mathrm{a}}\right)\right\},
\end{aligned}
$$

we can write a Galerkin scheme associated to (4.6): Find $\left(\omega_{\mathrm{B}, h}, p_{h}\right) \in \mathbf{Z}_{h}^{\mathrm{a}} \times \mathrm{Q}_{h}^{\mathrm{a}}$ such that

$$
\mathcal{A}^{\mathrm{a}}\left(\left(\omega_{\mathrm{B}, h}, p_{h}\right),\left(\theta_{h}, q_{h}\right)\right)=\mathcal{F}^{\mathrm{a}}\left(\theta_{h}, q_{h}\right) \quad \forall\left(\theta_{h}, q_{h}\right) \in \mathbf{Z}_{h}^{\mathrm{a}} \times \mathrm{Q}_{h}^{\mathrm{a}} .
$$


As in Section 3 we can compute continuous and discrete velocities using

$$
\boldsymbol{u}_{\mathrm{B}}=\kappa_{\mathrm{B}}\left(\mathbf{f}_{\mathrm{B}}-\sqrt{\nu} \operatorname{curl}_{\mathbf{a}} \omega_{\mathrm{B}}-\nabla p_{\mathrm{B}}\right) \quad \text { in } \quad \Omega_{\mathrm{B}}^{\mathrm{a}}, \quad \boldsymbol{u}_{\mathrm{D}}=\kappa_{\mathrm{D}}\left(\mathbf{f}_{\mathrm{D}}-\nabla p_{\mathrm{D}}\right) \quad \text { in } \quad \Omega_{\mathrm{D}}^{\mathrm{a}},
$$

and

$$
\boldsymbol{u}_{\mathrm{B}, h}=\kappa_{\mathrm{B}}\left(\mathcal{P}_{h} \mathbf{f}_{\mathrm{B}}-\sqrt{\nu} \operatorname{curl}_{\mathbf{a}} \omega_{\mathrm{B}, h}-\nabla p_{\mathrm{B}, h}\right) \quad \text { in } \Omega_{\mathrm{B}}^{\mathrm{a}}, \quad \boldsymbol{u}_{\mathrm{D}, h}=\kappa_{\mathrm{D}}\left(\mathcal{P}_{h} \mathbf{f}_{\mathrm{D}}-\nabla p_{\mathrm{D}, h}\right) \quad \text { in } \Omega_{\mathrm{D}}^{\mathrm{a}}
$$

On the other hand, the well-posedness analysis and error estimates for (4.9) can be established following the lines of Section 3 in combination with the following well-known result (cf. [28, Lemma 6.3], see also [8])

Lemma 4.1. There exists $C>0$, independent of $h$ and $\nu$, such that for all $\theta \in \mathrm{H}_{1}^{k+1}\left(\Omega^{\mathrm{a}}\right)$ :

$$
\left\|\theta-\Pi_{h} \theta\right\|_{\widetilde{\mathrm{H}}_{1}^{1}\left(\Omega^{\mathrm{a}}\right)} \leq C h^{k}\|\theta\|_{\mathrm{H}_{1}^{k+1}\left(\Omega^{\mathrm{a}}\right)},
$$

where $\Pi_{h}: \widetilde{\mathrm{H}}_{1}^{1}\left(\Omega^{\mathrm{a}}\right) \cap \mathrm{H}_{1}^{2}\left(\Omega^{\mathrm{a}}\right) \rightarrow \mathbf{Z}_{h}^{\mathrm{a}}$ is the Lagrange interpolator of a sufficiently smooth $\theta$.

Theorem 4.2 Convergence of the axisymmetric solution. Let us consider $\left(\omega_{\mathrm{B}}, p\right) \in \mathbf{Z}^{\mathrm{a}} \times \mathrm{Q}^{\mathrm{a}}$, and $\left(\omega_{\mathrm{B}, h}, p_{h}\right) \in \mathbf{Z}_{h}^{\mathrm{a}} \times \mathrm{Q}_{h}^{\mathrm{a}}$ to be the unique solutions of the continuous and discrete problems (4.6) and $(4.9)$, respectively. For $k \geq 1$, assume that $\omega_{\mathrm{B}} \in \mathrm{H}_{1}^{k+1}\left(\Omega_{\mathrm{B}}^{\mathrm{a}}\right), p \in \mathrm{H}_{1}^{k+1}\left(\Omega^{\mathrm{a}}\right), \mathbf{f}_{\mathrm{D}} \in \mathrm{H}_{1}^{k}\left(\Omega_{\mathrm{D}}^{\mathrm{a}}\right)^{2}$ and $\mathbf{f}_{\mathrm{B}} \in \mathrm{H}_{1}^{k}\left(\Omega_{\mathrm{B}}^{\mathrm{a}}\right)^{2}$. Then, there exist $C, \hat{C}>0$ independent of $h$ and $\nu$ such that

$$
\begin{aligned}
& \left\|\omega_{\mathrm{B}}-\omega_{\mathrm{B}, h}\right\|_{0, \Omega_{\mathrm{B}}^{\mathrm{a}}}+\left\|\sqrt{\nu} \operatorname{curl}\left(\omega_{\mathrm{B}}-\omega_{\mathrm{B}, h}\right)+\nabla\left(p_{\mathrm{B}}-p_{\mathrm{B}, h}\right)\right\|_{0, \Omega_{\mathrm{B}}}+\left\|\nabla\left(p-p_{h}\right)\right\|_{0, \Omega_{\mathrm{D}}^{\mathrm{a}}} \\
& +\left\|p-p_{h}\right\|_{0, \Omega^{\mathrm{a}}}+\left\|\boldsymbol{u}_{\mathrm{B}}-\boldsymbol{u}_{\mathrm{B}, h}\right\|_{0, \Omega_{\mathrm{B}}^{\mathrm{a}}}+\left\|\boldsymbol{u}_{\mathrm{D}}-\boldsymbol{u}_{\mathrm{D}, h}\right\|_{0, \Omega_{\mathrm{D}}^{\mathrm{a}}} \\
& \quad \leq C h^{k}\left(\left\|\omega_{\mathrm{B}}\right\|_{\mathrm{H}_{1}^{k+1}\left(\Omega_{\mathrm{B}}^{\mathrm{a}}\right)}+\|p\|_{\mathrm{H}_{1}^{k+1}\left(\Omega^{\mathrm{a}}\right)}+\left\|\mathbf{f}_{\mathrm{B}}\right\|_{\mathrm{H}_{1}^{k}\left(\Omega_{\mathrm{B}}^{\mathrm{a}}\right)}+\left\|\mathbf{f}_{\mathrm{D}}\right\|_{\mathrm{H}_{1}^{k}\left(\Omega_{\mathrm{D}}^{\mathrm{a}}\right)}\right),
\end{aligned}
$$

and

$$
\left\|\omega_{\mathrm{B}}-\omega_{\mathrm{B}, h}\right\|_{0, \Omega_{\mathrm{B}}^{\mathrm{a}}}+\left\|p-p_{h}\right\|_{0, \Omega^{\mathrm{a}}} \leq \hat{C} h^{k+1}\left(\left\|\omega_{\mathrm{B}}\right\|_{\mathrm{H}_{1}^{k+1}\left(\Omega_{\mathrm{B}}^{\mathrm{a}}\right)}+\|p\|_{\mathrm{H}_{1}^{k+1}\left(\Omega^{\mathrm{a}}\right)}\right)
$$

Proof. The first error estimate follows as a direct consequence of Lemma 4.1 and (4.10)-(4.11). The second estimate follows by a standard duality argument (see Theorem 3.6).

\section{NUMERICAL RESULTS}

The following set of examples serves to confirm numerically the convergence rates anticipated in Theorems 3.5, 3.6 and 3.7, and Theorem 4.2.

Test 1: Experimental convergence in 2D. We begin with a two-dimensional example where the Brinkman and Darcy subdomains $\Omega_{\mathrm{B}}=(0,1)^{2}, \Omega_{\mathrm{D}}=(0,1) \times(1,3 / 2)$ are separated by the segment $\Sigma=(0,1) \times\{1\}$. We choose arbitrary model parameters $\kappa_{\mathrm{D}}=0.02 \mathbf{I}, \kappa_{\mathrm{B}}=0.05 \mathbf{I}$, and $\nu=0.01$; and propose the following closed-form solutions to (2.1)-(2.5):

$$
\boldsymbol{u}_{\mathrm{B}}=\left(\begin{array}{c}
\sin (\pi x)^{2} \sin (\pi y)^{2} \cos (\pi y) \\
-\frac{1}{3} \sin (2 \pi x) \sin (\pi y)^{3}
\end{array}\right), \boldsymbol{u}_{\mathrm{D}}=\boldsymbol{u}_{\mathrm{B}} \times\left(\begin{array}{c}
0 \\
\frac{3}{2}-y
\end{array}\right), \boldsymbol{\omega}_{\mathrm{B}}=\sqrt{\nu} \operatorname{curl} \boldsymbol{u}_{\mathrm{B}}, p=\left(x-\frac{1}{2}\right)^{3}-\left(y-\frac{3}{2}\right)^{3},
$$

which satisfy exactly the boundary and interface conditions (2.6)-(2.10). Notice that $\boldsymbol{u}_{\mathrm{D}}$ is not divergence free, but $g_{\mathrm{D}}$ (and analogously the forcing terms $\mathbf{f}_{\mathrm{B}}, \mathbf{f}_{\mathrm{D}}$ ) are constructed from the manufactured solutions above. Two families of successively refined unstructured meshes for $\Omega_{\mathrm{B}}$ and $\Omega_{\mathrm{D}}$ are generated, matching on the interface as required by the conformity of the global pressure approximation. We stress that the discrete velocities are obtained as postprocess from the discrete Brinkman vorticity and discrete global pressure. Moreover, the zero-mean condition enforcing the uniqueness of the global pressure is implemented using a scalar Lagrange multiplier (adding one row and one column to the matrix system that solves for $\boldsymbol{\omega}_{\mathrm{B}, h}$ and $p_{h}$ ). All linear systems are solved using the direct method 
TABLE I. Test 1. Experimental accuracy of the proposed finite element method against manufactured exact solutions. Convergence achieved using a scheme of increasing order $k \in\{1,2,3\}$ (from top to bottom).

\begin{tabular}{|c|c|c|c|c|c|c|c|c|c|c|c|c|}
\hline$h$ & $e_{0}^{\boldsymbol{u}_{\mathrm{B}}}$ & rate & $e_{0}^{\boldsymbol{u}_{\mathrm{D}}}$ & rate & $e_{0}^{\boldsymbol{\omega}_{\mathrm{B}}}$ & rate & $e_{1}^{\left(\boldsymbol{\omega}_{\mathrm{B}}, 1\right.}$ & rat & $e_{1}^{p_{\mathrm{D}}}$ & rate & $e_{0}^{p}$ & rat \\
\hline \multicolumn{13}{|c|}{$(k=1)$} \\
\hline 707 & 2755 & - & 0.1084 & - & 0.0164 & - & 0.3901 & - & 0.3235 & - & 0.0700 & - \\
\hline 500 & 1308 & 0.90 & 7 & 1.47 & & 1.41 & 8 & 0.97 & & 0.97 & .0407 & 1.6 \\
\hline 26 & 0677 & 1 & $x$ & 0.84 & & 2.0 & & 1 & & & & 2.0 \\
\hline 99 & 038 & & & & & & & & & & & 2.02 \\
\hline 4 & & & & & & & & & & & & 1.94 \\
\hline 8 & 1 & & & & & & & & & & 03 & 1.99 \\
\hline 5 & 0052 & 1 & 9 & & & 1. & 0 & & & & $60 \mathrm{e}-5$ & 2.02 \\
\hline 3 & 0.0026 & 1. & 0.0015 & 1.08 & e-6 & 2.0 & 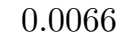 & 9 & & .09 & $97 \mathrm{e}-5$ & 2.02 \\
\hline & 0013 & 1 & 0.0007 & 1.1 & e-6 & 1.9 & 2 & 8 & & .15 & $95 \mathrm{e}-6$ & 2.01 \\
\hline 4 & 0.0007 & 1.10 & 0.0 & 1.09 & e-7 & 1.99 & 6 & 11 & & 1.00 & $1.26 \mathrm{e}-6$ & 1.99 \\
\hline \multicolumn{13}{|c|}{$(k=$} \\
\hline 07 & 0.2157 & - & & - & & - & 0 & - & & 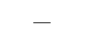 & & - \\
\hline 500 & 0.0977 & 08 & 9 & 2.07 & 5 & 1.9 & & & & .25 & 20 & 3.2 \\
\hline 326 & 0.0466 & 1 & 0.0198 & 1.75 & 03 & 3.0 & 5 & 2 & 84 & .07 & .0006 & 3.12 \\
\hline 99 & 0.0153 & 2 & & & & 2.9 & & & & & & .00 \\
\hline & & & 3 & & -5 & 2.87 & & & & 7 & e- 6 & 2.91 \\
\hline & & & & & & & & & & & & 3.01 \\
\hline & & & & & & & & & & & & 3.0 \\
\hline 3 & 27 & 2 & & 8 & & 3. & & & & 2.01 & e- 8 & $3.0 \mathrm{C}$ \\
\hline 07 & $.73 e-5$ & 2.10 & $1.49 \mathrm{e}-5$ & 2.17 & e-9 & 2.9 & 1 & 2.0 & 3.8 & 1.89 & $02 \mathrm{e}-8$ & 3.00 \\
\hline 04 & $4.55 \mathrm{e}-6$ & 2.03 & $5.36 \mathrm{e}-6$ & 2.09 & $.02 \mathrm{e}-9$ & 2.49 & $3.91 \mathrm{e}-6$ & 1.99 & $9.45 \mathrm{e}-7$ & 1.98 & $4.24 \mathrm{e}-9$ & 3.01 \\
\hline \multicolumn{13}{|c|}{$(k=3)$} \\
\hline & & 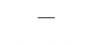 & & - & & & & & & - & & - \\
\hline & 0.030 & & & & & & & & & & & 1.96 \\
\hline & 0.0054 & & & & & 4. & & & & 3. & 01 & 4 \\
\hline & 0.0009 & 3.1 & 0.0004 & 3. & e-5 & 4. & 7 & 3. & 03 & 3.30 & 01e-6 & 4.2 \\
\hline & .0001 & 2 & $93 e-5$ & 28 & $66 e-7$ & 4. & -5 & 3 & -5 & 3.23 & e-7 & 4.2 \\
\hline 048 & $1.65 \mathrm{e}-5$ & 3 & $1.17 \mathrm{e}-5$ & 2.85 & $17 \mathrm{e}-8$ & 4.13 & $7.54 \mathrm{e}-6$ & 3.20 & $39 \mathrm{e}-6$ & 3.19 & $10 \mathrm{e}-8$ & 4.18 \\
\hline 025 & $2.21 \mathrm{e}-6$ & 3.02 & 3e- 6 & 3.24 & -9 & 4.10 & & .14 & & 3.02 & $.7 \mathrm{e}-10$ & 4.10 \\
\hline 013 & $.95 \mathrm{e}-7$ & 3 & $1.85 \mathrm{e}-7$ & 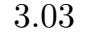 & & 4.0 & & 3 & & 3.00 & $5 \mathrm{e}-11$ & 4.0 \\
\hline & & & & 2. & & 3. & & & & 3.10 & $2.2 \mathrm{e}-12$ & 3.7 \\
\hline 0.004 & $4.01 \mathrm{e}-9$ & 2.99 & $1.86 \mathrm{e}-9$ & 2.99 & $8.2 \mathrm{e}-13$ & 4.31 & $1.16 \mathrm{e}-9$ & 2.95 & $1.8 \mathrm{e}-10$ & 2.87 & $1.4 \mathrm{e}-13$ & 3.93 \\
\hline
\end{tabular}

MUMPS. Errors between the exact and approximate solutions are measured in the norms involved in the convergence analysis of Section 2, and will adopt the following notation

$$
e_{1}^{\left(\boldsymbol{\omega}_{\mathrm{B}}, p_{\mathrm{B}}\right)}=\left\|\sqrt{\nu} \operatorname{curl}\left(\boldsymbol{\omega}_{\mathrm{B}}-\boldsymbol{\omega}_{\mathrm{B}, h}\right)+\nabla\left(p_{\mathrm{B}}-p_{\mathrm{B}, h}\right)\right\|_{0, \Omega_{\mathrm{B}}}, \quad e_{1}^{p_{\mathrm{D}}}=\left\|\nabla\left(p_{\mathrm{D}}-p_{\mathrm{D}, h}\right)\right\|_{0, \Omega_{\mathrm{D}}},
$$

whereas $e_{0}^{s}$ will denote the $L^{2}$-norm of the error associated to the generic quantity $s$. The obtained error history is reported in Table I. The asymptotic $O\left(h^{k}\right)$ decay of the error observed for each field (except for the Brinkman vorticity and global pressure in their $L^{2}$-norms, which exhibit a decay of $O\left(h^{k+1}\right)$ ), indicates an overall optimal convergence of the proposed method as predicted by Theorems 3.5, 3.6, and 3.7. Sample approximate solutions generated with the lowest order method are displayed in Figure 2, showing accurate profiles, also near the interface.

Test 2: Cracked porous media. Our next test focuses on the simulation of flow in fractured porous structures, and the test configuration has been adapted from [2]. A box of two porous ma- 

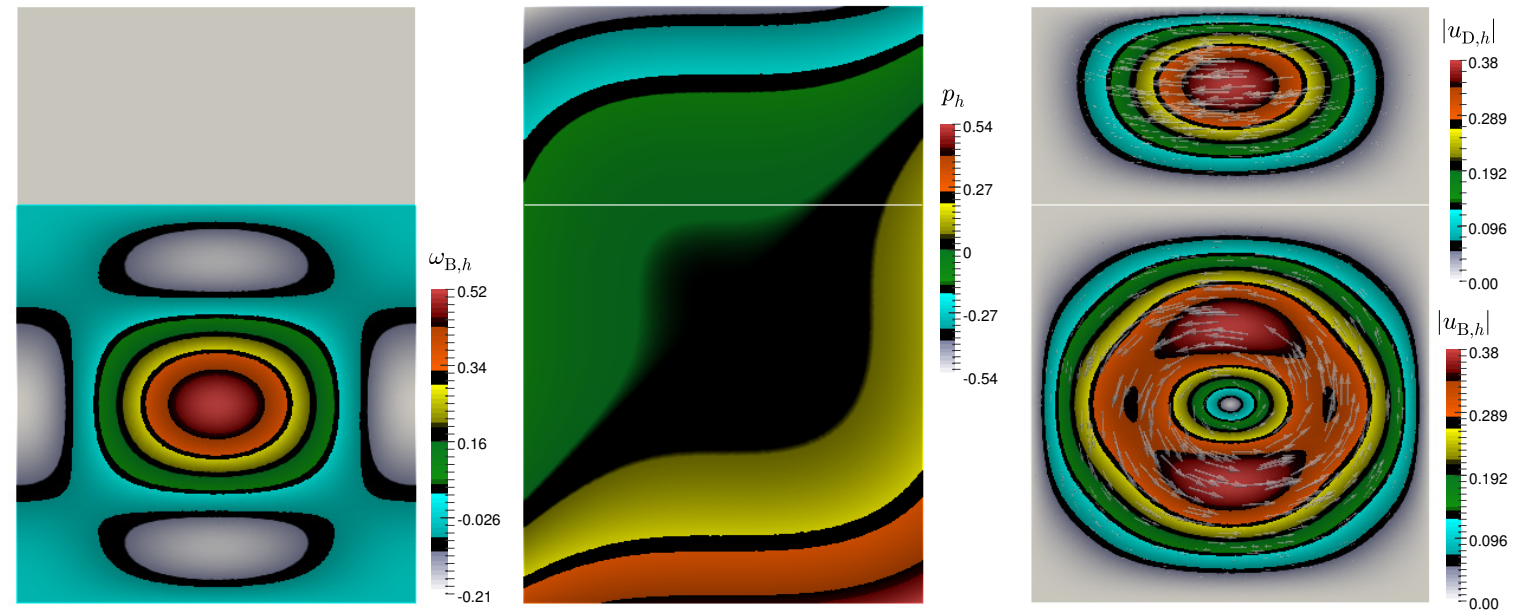

FIG. 2. Test 1. Numerical solution of the Brinkman-Darcy coupled problem, generated with the lowest order method. Brinkman vorticity (left), global pressure (centre), and postprocessed velocities (right).

terials having different volume fractions of calcarenite and sand is considered, where the interface between the Brinkman and Darcy subdomains is a smoothed "V-shaped" surface (see also $[9,12]$ ). The domain size is now $[0,1.5] \times[0,0.2] \times[0,1]$, the Darcy subdomain is located below $\Sigma$, and external forces on both domains correspond to gravity, and a smooth flow rate in the $x$-direction $\mathbf{f}_{\mathrm{B}}=\mathbf{f}_{\mathrm{D}}=\left(0.1 \cos ^{2}(\pi x z), 0,-0.98\right)^{T}$. The permeabilities and viscosity are set to $\kappa_{\mathrm{B}}=5 \mathbf{I}, \kappa_{\mathrm{D}}=1 \mathbf{I}$, and $\nu=0.001$. The unstructured tetrahedral mesh consists of approximately 1313372 elements and 241250 vertices. We use the same strategy to impose zero-mean pressure as in the previous test, but now the linear systems are inverted with the BICGSTAB Krylov solver, and the discrete Brinkman vorticity is approximated using Nédélec elements with $k=1$. Such a spatial resolution and the chosen finite element family implies that the matrix system has 408503 unknowns. The method from [2] entails a discrete problem with 4173527 (more than 10 times the cost of the present scheme). The generated numerical solutions are presented in Figure 3, and one expects continuity of the normal velocities across the interface (observed in the streamlines on the bottom right panel), very regular pressure profiles and continuous across the interface (that we see in the top right panel). The flow patterns in the Brinkman domain are however not expected to be as uniform as in [2], since the problem configuration in our case assumes a non-constant flow rate. This condition also implies that now the flow is higher in the larger Darcy domain.

Test 3: Accuracy assessment in an axisymmetric enclosure. We finally turn to the verification of the convergence analysis in the axisymmetric case, for which we construct a cylindrical domain of height 4 , representing a simplified oil filter (see a similar test in [5]). We write the problem and its discretisation using a half cross-section of the domain, with minimum and maximum radii 0.5 and 2 , respectively. The Brinkman subdomain (located on the upper part of the filter) is separated from the Darcy domain by a curved interface $\Sigma^{\mathrm{a}}$ parametrised, in the meridional axisymmetric coordinates, as $(r, z)=(t, 2+0.2 t+0.1 t \cos [6 \pi t-3 \pi])$, with $t \in(0,2)$. The permeabilities in each domain are constant $\kappa_{\mathrm{B}}=500 \mathbf{I}, \kappa_{\mathrm{D}}=100 \mathbf{I}$, and the fluid viscosity is $\nu=0.01$. We construct smooth forcing terms representing an external motion of the filter $\mathbf{f}_{\mathrm{B}}=\left(0,-\nu r \sin ^{2}(\pi r z)\right)^{T}, \mathbf{f}_{\mathrm{D}}=\left(0, \nu z^{2} \cos (2 \pi r z)\right)^{T}$, and choose $g_{\mathrm{D}}=-\nu \sin (\pi r) \sin (\pi z)$. Interface and boundary conditions are imposed as in (4.3)-(4.5), and in the absence of a closed-form solution to the problem we generate a reference numerical solution with a method of order $k=3$, and using a highly refined mesh for the axisymmetric domain. Then we produce a sequence of coarser meshes and obtain approximate solutions using (4.9) with $k=2$. We compute errors against the reference fine solution and collect the result of the convergence history in Table II. Again we evidence optimal rates of convergence, this time according to Theorem 4.2. We also depict the numerical solutions and extrude into the cylindrical domain the approximate Brinkman vorticity, global pressure and individual velocities (see Figure 4). For a given spatial resolution, the 

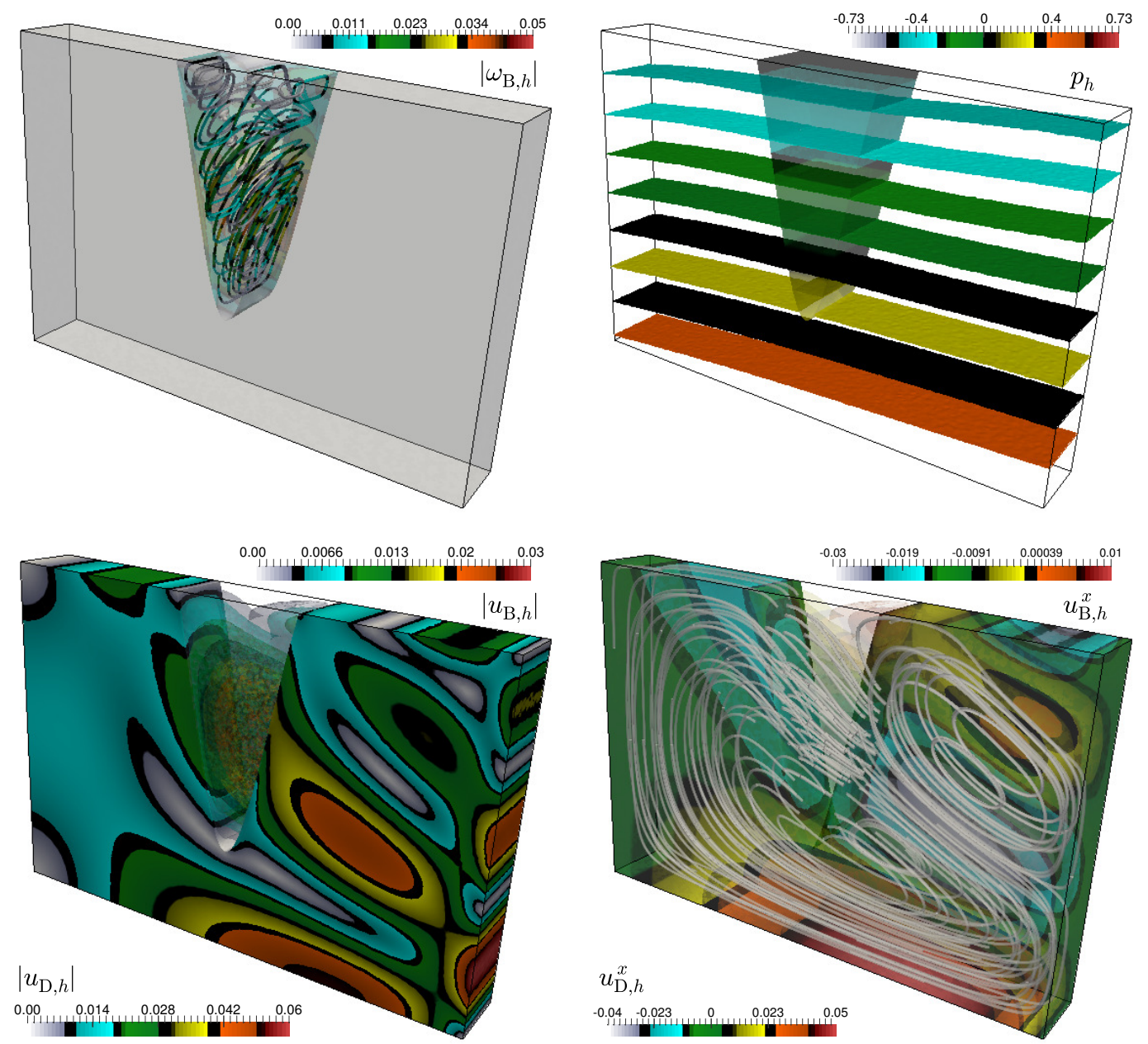

FIG. 3. Test 2. Numerical solution of the Brinkman-Darcy coupled problem, generated with the lowest order method. Brinkman vorticity magnitude and streamlines (top left), iso-surfaces of the global pressure (top right), and postprocessed velocities (magnitudes on the bottom left, and example of the $x$-component and streamlines on bottom right).

method with $k=1$ results in a matrix system with 38103 degrees of freedom, and if the polynomial order is $k=2$ then the matrix system has 151232 unknowns. Other schemes may be much more expensive, as for example the methods for axisymmetric Stokes-Darcy problems proposed in [15], which for the same mesh involves 301820 degrees of freedom for the scheme that uses Taylor-Hood elements for the approximation of velocity and pressure in the Stokes domain, or 275216 degrees of freedom for the one using the MINI-element in that same region.

Concluding remarks. We recall that as the discrete global pressure is $\mathrm{H}^{1}(\Omega)$ (or $\mathrm{H}^{1}\left(\Omega^{\mathrm{a}}\right)-$ ) conforming, the meshes for the Brinkman and Darcy subdomains have been assumed to match on the interface. In order to relax this condition, one could devise appropriate extensions using domain decomposition or a discontinuous Galerkin counterpart of (3.3), which could be quite useful in e.g. subsurface flow applications. Another extension currently under study is the imposition of stress-free boundary conditions (which are not straightforward in the context of vorticity-based formulations). These ideas are not explored here, but they certainly deserve further investigation.

Acknowledgement. V. Anaya was partially supported by CONICYT-Chile through FONDECYT project 11160706 and DIUBB through project $165608-3 / \mathrm{R}$; R. Ruiz-Baier acknowledges the support by the EPSRC through the project EP/R00207X/1. 

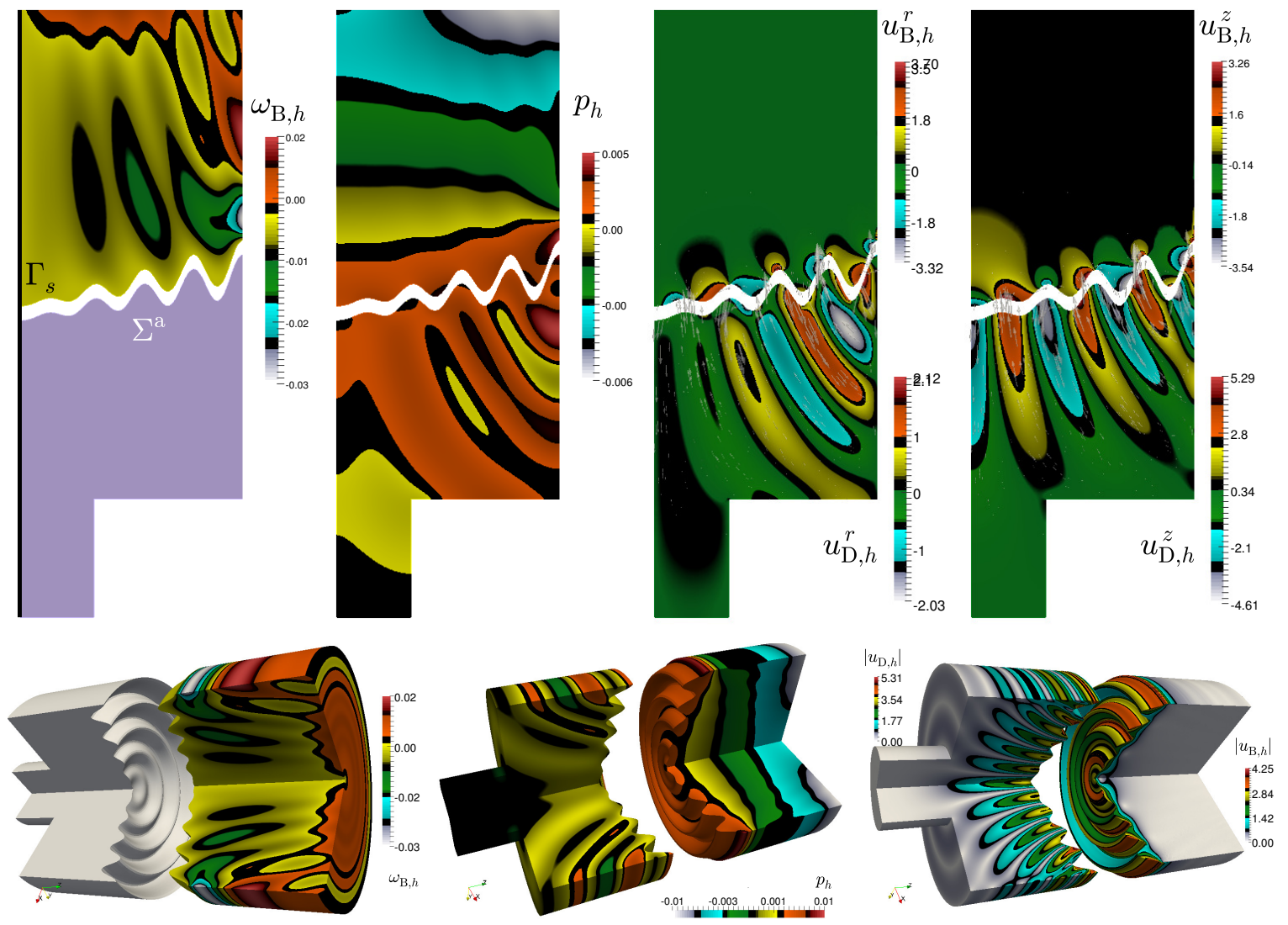

FIG. 4. Test 3. Numerical solution of the axisymmetric Brinkman-Darcy coupled problem, generated with the second order method. Brinkman vorticity and sketch of curved interface and symmetry axis (top left), distribution of global pressure (top, second panel), and radial and vertical components of the postprocessed velocities (top third and right figures). The bottom row shows different views of the axisymmetric solutions extruded to the three-dimensional domain (only an angle of $3 \pi / 4$ is plotted for visualisation purposes).

TABLE II. Test 3. Error history associated to the finite element method with order $k=2$, in an axisymmetric setting. Errors measured in the weighted norms (denoted with subscript $a$ ), and computed against a reference fine-mesh solution obtained with a higher order method.

\begin{tabular}{ccccccccccccc}
\hline$h$ & $e_{0, a}^{\boldsymbol{u}_{\mathrm{B}}}$ & rate & $e_{0, a}^{\boldsymbol{u}_{\mathrm{D}}}$ & rate & $e_{0, a}^{\boldsymbol{\omega}_{\mathrm{B}}}$ & rate & $e_{1, a}^{\left(\boldsymbol{\omega}_{\mathrm{B}}, p_{\mathrm{B}}\right)}$ & rate & $e_{1, a}^{p_{\mathrm{D}}}$ & rate & $e_{0, a}^{p}$ & rate \\
\hline 0.7311 & 0.4045 & - & 0.4024 & - & 0.0281 & - & 0.1012 & - & 0.0615 & - & 0.0252 & - \\
0.5193 & 0.1814 & 2.13 & 0.2062 & 1.97 & 0.0120 & 2.45 & 0.0429 & 1.91 & 0.0452 & 2.06 & 0.0061 & 2.92 \\
0.3242 & 0.0802 & 1.85 & 0.0689 & 1.94 & 0.0027 & 2.82 & 0.0201 & 1.94 & 0.0151 & 1.93 & 0.0028 & 2.95 \\
0.2141 & 0.0322 & 1.95 & 0.0281 & 2.02 & 0.0008 & 2.99 & 0.0083 & 1.96 & 0.0077 & 1.96 & 0.0005 & 2.98 \\
0.1162 & 0.0080 & 1.98 & 0.0064 & 1.96 & $1.10 \mathrm{e}-4$ & 3.00 & 0.0025 & 1.94 & 0.0022 & 1.94 & $8.31 \mathrm{e}-5$ & 2.92 \\
0.0533 & 0.0020 & 1.95 & 0.0016 & 2.01 & $1.71 \mathrm{e}-5$ & 2.97 & 0.0006 & 1.97 & 0.0004 & 1.95 & $1.22 \mathrm{e}-5$ & 2.99 \\
0.0316 & 0.0006 & 2.04 & 0.0005 & 2.04 & $2.18 \mathrm{e}-6$ & 2.93 & 0.0002 & 2.03 & $7.98 \mathrm{e}-5$ & 1.96 & $1.58 \mathrm{e}-6$ & 3.03 \\
0.0150 & $1.16 \mathrm{e}-4$ & 1.98 & $1.04 \mathrm{e}-4$ & 1.98 & $2.80 \mathrm{e}-7$ & 2.87 & $4.55 \mathrm{e}-5$ & 1.93 & $2.32 \mathrm{e}-5$ & 2.08 & $2.01 \mathrm{e}-7$ & 2.98 \\
0.0079 & $3.41 \mathrm{e}-5$ & 2.01 & $2.71 \mathrm{e}-5$ & 1.95 & $4.79 \mathrm{e}-8$ & 2.95 & $1.21 \mathrm{e}-5$ & 2.02 & $5.47 \mathrm{e}-6$ & 1.94 & $1.95 \mathrm{e}-8$ & 2.95 \\
0.0042 & $8.63 \mathrm{e}-6$ & 1.97 & $7.01 \mathrm{e}-6$ & 1.93 & $1.35 \mathrm{e}-8$ & 2.98 & $2.72 \mathrm{e}-6$ & 1.97 & $1.67 \mathrm{e}-6$ & 1.96 & $1.90 \mathrm{e}-9$ & 2.96 \\
\hline
\end{tabular}

\section{REFERENCES}

1. A. Alonso, And A. Valli, An optimal domain decomposition preconditioner for low-frequency time harmonic Maxwell equations. Math. Comp., 68 (1999) 607-631. 
2. M. Alvarez, G.N. Gatica, And R. Ruiz-Baier, Analysis of a vorticity-based fully-mixed formulation for the 3D Brinkman-Darcy problem. Comput. Methods Appl. Mech. Engrg., 307 (2016) 68-95.

3. M. Alvarez, G.N. Gatica, And R. Ruiz-Baier, A posteriori error analysis of a fully-mixed formulation for the Brinkman-Darcy problem. Calcolo, 54(4) (2017) 1491-1519.

4. V. Anaya, D. Mora, R. Oyarzúa, And R. Ruiz-Baier, A priori and a posteriori error analysis for a mixed scheme for the Brinkman problem. Numer. Math., 133 (2016) 781-817.

5. V. Anaya, D. Mora, C. Reales, And R. Ruiz-Baier, Stabilized mixed approximation of axisymmetric Brinkman flows. ESAIM Math. Model. Numer. Anal., 49(3) (2015) 855-874.

6. V. Anaya, D. Mora, And R. Ruiz-Baier, Pure vorticity formulation and Galerkin discretization for the Brinkman equations. IMA J. Numer. Anal., 37(4) (2017) 2020-2041.

7. T. Arbogast, and D.S. Brunson, A computational method for approximating a Darcy-Stokes system governing a vuggy porous medium. Comput. Geosci., 11 (2007) 207-218.

8. Z. Belhachmi, C. Bernardi, And S. Deparis, Weighted Clément operator and application to the finite element discretization of the axisymmetric Stokes problem. Numer. Math., 105 (2002) 217-247.

9. C. Bernardi, F. Hecht, and F.Z. Nouri, A new finite-element discretization of the Stokes problem coupled with the Darcy equations. IMA J. Numer. Anal., 30(1) (2010) 61-93.

10. C. Bernardi, F. Hecht, and O. Pironneau, Coupling Darcy and Stokes equations for porous media with cracks. ESAIM Math. Model. Numer. Anal., 39(1) (2005) 7-35.

11. M. BRAACK, AND F. SCHIEWECK, Equal-order finite elements with local projection stabilization for the Darcy-Brinkman equations. Comput. Methods Appl. Mech. Engrg., 200(9-12) (2011) 1126-1136.

12. J. Camaño, G.N. Gatica, R. Oyarzúa, R. Ruiz-Baier, and P. Venegas, New fully-mixed finite element methods for the Stokes-Darcy coupling. Comput. Methods Appl. Mech. Engrg., 295 (2015) 362395.

13. M. Discacciati, AND R. OyarzúA, A conforming mixed finite element method for the NavierStokes/Darcy coupled problem. Numer. Math., 135(2) (2017) 571-606.

14. F. El Chami, G. Mansour, and T. Sayah, Error studies of the coupling Darcy-Stokes system with velocity-pressure formulation. Calcolo, 49(2) (2012) 73-93.

15. V.J. ERvin, Approximation of coupled Stokes-Darcy flow in an axisymmetric domain. Comput. Methods Appl. Mech. Engrg., 258(1) (2013) 96-108.

16. V.J. Ervin, E.W. JEnkins, AND S. Sun, Coupled generalized nonlinear Stokes flow with flow through a porous medium. SIAM J. Numer. Anal., 47(2) (2009) 929-952.

17. G.N. Gatica, L.F. Gatica, and A. MÁrquez, Analysis of a pseudostress-based mixed finite element method for the Brinkman model of porous media flow. Numer. Math., 126(4) (2014) 635-677.

18. G.N. Gatica, A. Márquez, And S. MedDahi, Analysis of the coupling of primal and dual-mixed finite element methods for a two-dimensional fluid-solid interaction problem. SIAM J. Numer. Anal., 45(5) (2007) 2072-2097.

19. G.N. Gatica, S. Meddahi, and R. Oyarzúa, A conforming mixed finite-element method for the coupling of fluid flow with porous media flow. IMA J. Numer. Anal., 29(1) (2009) 86-108.

20. G.N. Gatica, R. Oyarzúa, and F.J. Sayas, Convergence of a family of Galerkin discretizations for the Stokes-Darcy coupled problem. Numer. Methods PDEs, 27(3) (2011) 721-748.

21. G. Kanschat, And B. Rivière, A strongly conservative finite element method for the coupling of Stokes and Darcy flow. J. Comput. Phys., 229 (2010) 5933-5943.

22. U. LACIS, AND S. BAGHERI, A framework for computing effective boundary conditions at the interface between free fluid and a porous medium. J. Fluid Mech., 812 (2017) 866-889.

23. W.J. Layton, F. Schieweck, And I. Yotov, Coupling fluid flow with porous media flow. SIAM J. Numer. Anal., 40(6) (2003) 2195-2218.

24. M. Lesinigo, C. D'Angelo, and A. Quarteroni, A multiscale Darcy-Brinkman model for fluid flow in fractured porous media. Numer. Math., 117(4) (2011) 717-752.

25. Y. Mabrouki, S. Mani Aound, And J. Satouri, Spectral discretization of Darcy equations coupled with Stokes equations by vorticity-velocity-pressure formulations. Numer. Methods PDEs, 33 (2017) 1628-1651. 
26. A. Márquez, S. Meddahi, and F.J. Sayas, Strong coupling of finite element methods for the StokesDarcy problem. IMA J. Numer. Anal., 35(2) (2015) 969-988.

27. G. Matthies, and L. Tobiska, Mass conservation of finite element methods for coupled flow-transport problems. Int. J. Comput. Sci. Math., 1 (2007) 293-307.

28. B. Mercier, And G. RAugel, Resolution d'un problème aux limites dans un ouvert axisymétrique par éléments finis en $r$, $z$ et séries de Fourier en $\theta$. RAIRO Anal. Numér., 16 (1982) 405-461.

29. A. Mikelic, AND W. JäGER, On the interface boundary conditions by Beavers, Joseph and Saffman. SIAM J. Appl. Math., 60(4) (2000) 1111-1127.

30. B. RIVIÈre, Analysis of a discontinuous finite element method for the coupled Stokes and Darcy problems. J. Sci. Comput., 22-23 (2005) 479-500. 